Supporting Information

\title{
Use of Standard Addition to Quantify in-situ FTIR Reaction Data
}

George Hutchinson ${ }^{\mathrm{a}}$, Calum D. M. Welsh ${ }^{\mathrm{b}}$ and Jordi Burés*a

\begin{abstract}
a - The University of Manchester, Department of Chemistry, Oxford Road, Manchester, M13 9PL, UK b - Mettler-Toledo Ltd., 64 Boston Road, Beaumont Leys, Leicester, LE4 1AW

jordi.bures@manchester.ac.uk
\end{abstract}

\section{Table of Contents}

1. General information

2. Description of the experimental set up

3. Verification of the linear relationship between concentration and FTIR signal S-4

4. Derivation of Equations 1 and $2 \quad$ S-6

5. Application of the standard addition method to the organocatalytic $\alpha$-selenylation of aldehydes S-8

6. Requirements of the standard addition method $\quad$ S-14

$\begin{array}{ll}\text { 7. References } & \text { S-14 }\end{array}$

8. Appendix 1 - Data for figures S2 and S3 S-15

9. Appendix 2 - Data for figures S4 and S5 S-16

10. Appendix 3 - Data for figures $1,2,3,4, S 6, S 7, S 8$ and $S 9$

11. Appendix 4 - Data for figures 5, S11 and S12 S-24 


\section{General Information}

Unless otherwise specified, reagents and solvents were used as purchased from Merck, Alfa-Aesar and Fisher Scientific. $\mathrm{CDCl}_{3}$ was dried over $4 \AA$ activated molecular sieves and stored under nitrogen prior to use. The amine catalyst, (S)- $\alpha, \alpha$-bis[3,5-bis(trifluoromethyl)phenyl]-2-pyrrolidinemethanol trimethylsilyl ether, was purified by flash column chromatography $\left(100 \% \mathrm{CH}_{2} \mathrm{Cl}_{2}\right)$ to remove any deprotected alcohol. $\mathrm{N}$-(Phenylseleno)phthalimide (NPSP) was recrystallized from $\mathrm{CHCl}_{3}$, washed with cold isopropyl alcohol and stored at $-18{ }^{\circ} \mathrm{C}$ under nitrogen. The recrystallized NPSP was found to be $98 \%$ pure by NMR, and this has been accounted for in all concentration calculations. Isovaleraldehyde was freshly distilled under vacuum prior to use.

All FTIR spectra were taken with a Mettler Toledo ReactIR 15 equipped with a $\mathrm{LN}_{2} \mathrm{MCT}$ detector and a $9.5 \mathrm{~mm} \mathrm{AgX} \mathrm{Fibre} \mathrm{DiComp} \mathrm{probe} \mathrm{at} 4 \mathrm{~cm}^{-1}$ resolution. The spectra were recorded every $45 \mathrm{~s}$ and each was comprised of 122 scans. The FTIR data underwent $2^{\text {nd }}$ derivative processing using the standard function in the Mettler-Toledo iCIR software. This function applies a 7-point Savitzky-Golay filter and an inversion. The $\mathrm{N}$-(phenylseleno)phthalimide (NPSP) was monitored using the height of the signal at $862 \mathrm{~cm}^{-1}$ relative to zero. The height of the signal at $1036 \mathrm{~cm}^{-1}$ relative to a single point baseline at $1058 \mathrm{~cm}^{-1}$ was used to monitor the concentration of phthalimide (NHP). A minimum of 5 data points before and after each addition were taken to ensure a reliable average. The NPSP and NHP were each added in two portions and the average of these two calibrations was reported. The average temperatures during the reactions were recorded by the FTIR probe.

All NMR spectra were recorded on a Bruker AVIII HD $400 \mathrm{MHz}$ spectrometer with BBO prodigy probe. The ${ }^{1} \mathrm{H}$ NMR chemicals shifts $(\delta)$ are quoted in ppm relative to residual solvent peaks (for $\mathrm{CDCl}_{3}$, given in ppm: 7.26). Quantitative ${ }^{1} \mathrm{H}$ NMR (qNMR) was performed with $a d_{1}=25 \mathrm{~s}$ to ensure all species had fully relaxed. The analysis of reaction yield by qNMR used 1,1,2,2-tetrachloroethane as the internal standard. 


\section{Description of the experimental set up}

We set up the ReactIR such that any movement of the probe during the reactions was minimized and the detector was isolated from vibrations. We inserted the ReactIR probe into a capped vial with a presplit septum and stirrer bar. After we had injected the reagents, we sealed the system with Parafilm to minimize any possible evaporation.
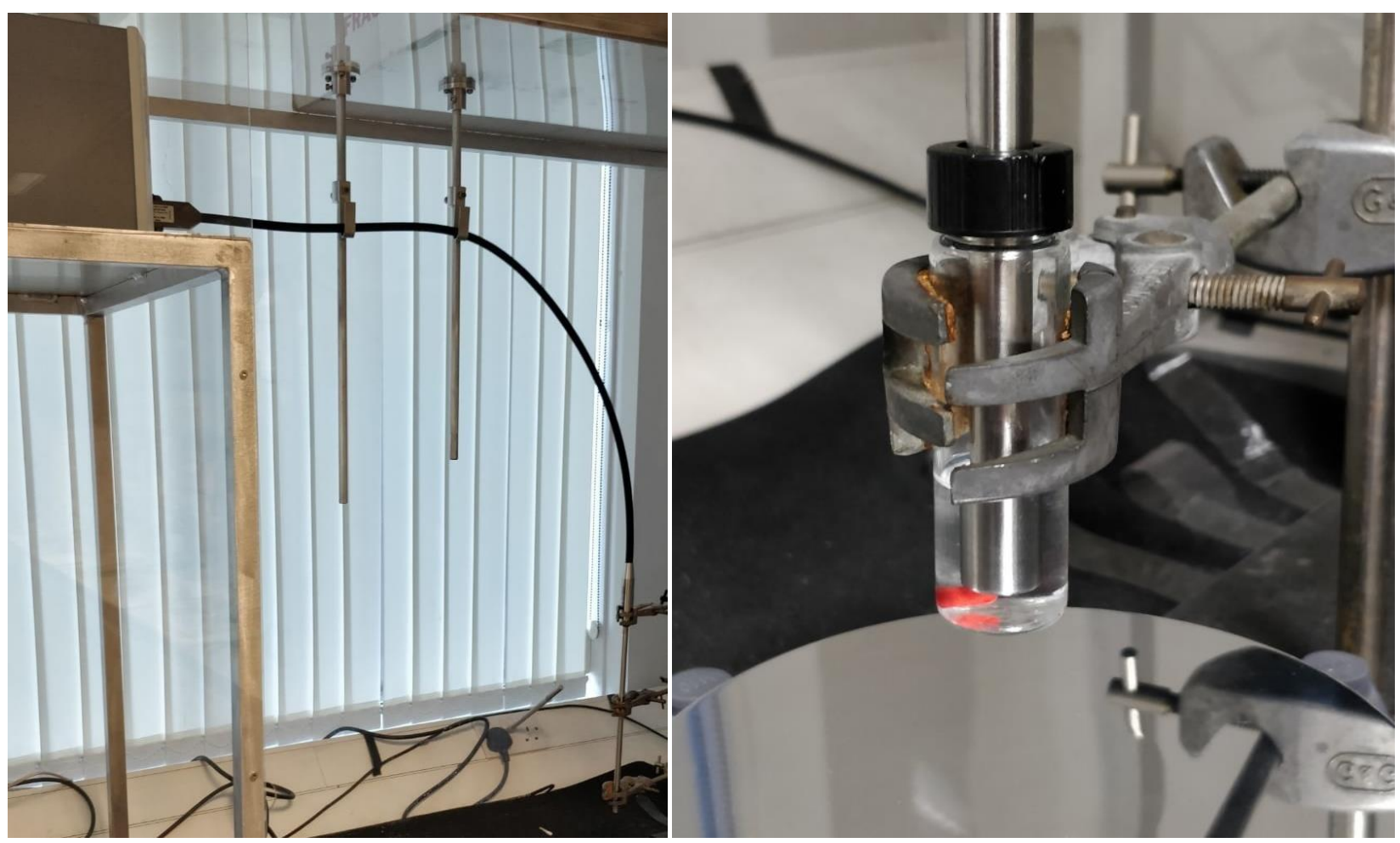

Figure S1 - Photos of the ReactIR set up. 


\section{Verification of the linear relationship between concentration and FTIR signal}

We added a solution of $\mathrm{N}$-(phenylseleno)phthalimide in $\mathrm{CDCl}_{3}(0.035 \mathrm{M}, 2.5 \mathrm{~mL}$ ) to a vessel containing the FTIR probe, a stirrer bar and $\mathrm{CDCl}_{3}(1.5 \mathrm{~mL})$ in five portions ( $500 \mu \mathrm{L}$ each). This covered the range of concentration possible during the organocatalytic $\alpha$-selenylation of aldehydes reaction. We plotted the measured signal intensity, at $862 \mathrm{~cm}^{-1}$, against the calculated concentration to demonstrate the linear relationship. The data is tabulated in Appendix 1 (S-15).

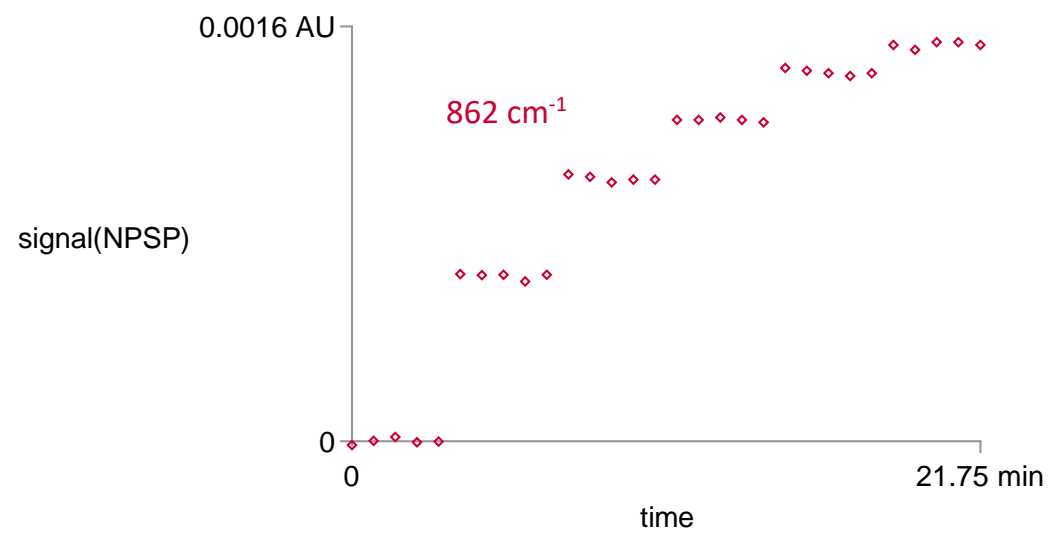

Figure S2 - The NPSP signal grows non-linearly with each addition as the volume is increased.

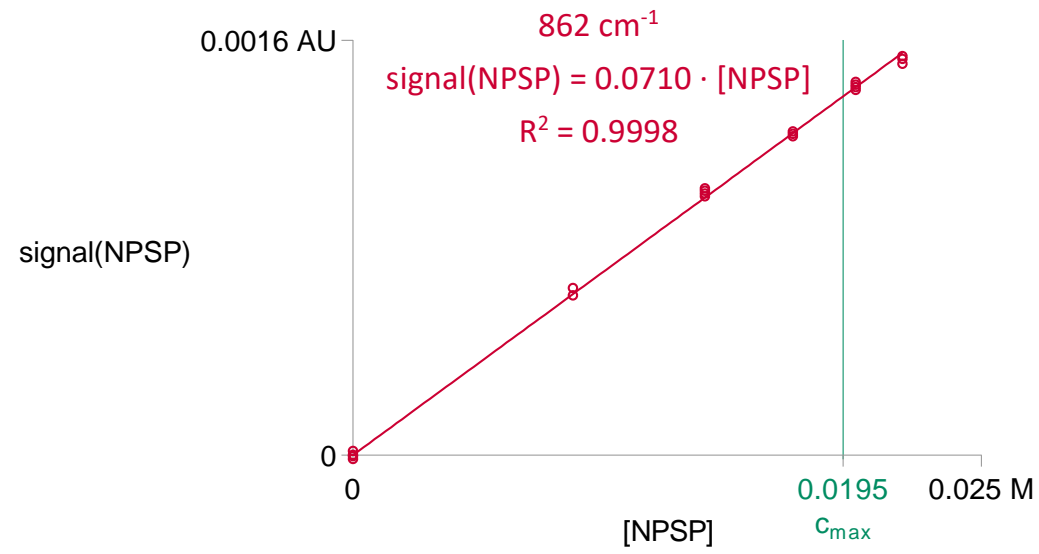

Figure S3 - The NPSP signal is proportional to the concentration. 
We added a solution of phthalimide in $\mathrm{CDCl}_{3}(0.04 \mathrm{M}, 2.5 \mathrm{~mL})$ to a vessel containing the FTIR probe, a stirrer bar and $\mathrm{CDCl}_{3}(1.5 \mathrm{~mL})$ in five portions (500 $\mu \mathrm{L}$ each). This covered the range of concentration possible during the organocatalytic $\alpha$-selenylation of aldehydes reaction. We plotted the measured signal intensity at $1036 \mathrm{~cm}^{-1}$ against the calculated concentration to demonstrate the linear relationship. The data is tabulated in Appendix 2 (S-16).

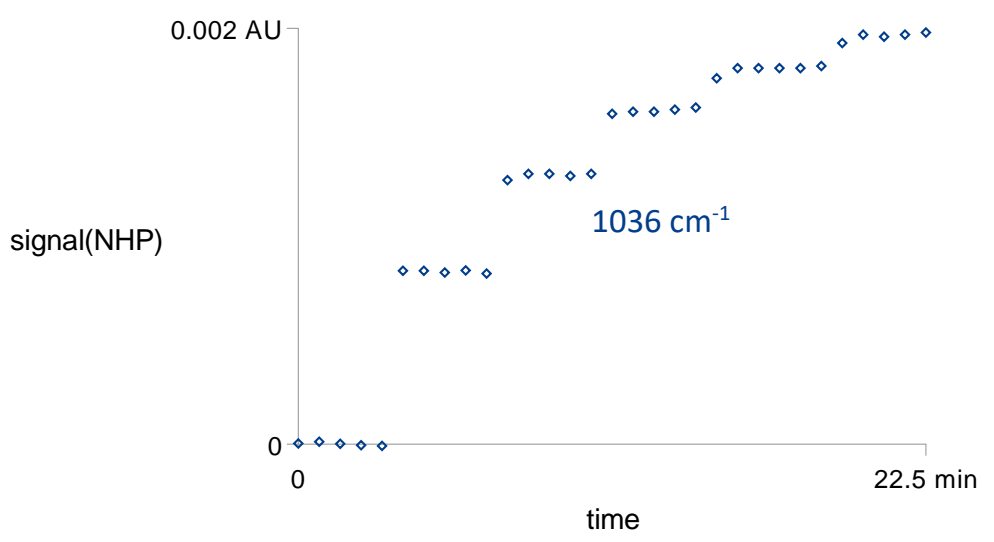

Figure S4 - The NHP signal grows non-linearly with each addition as the volume is increased.

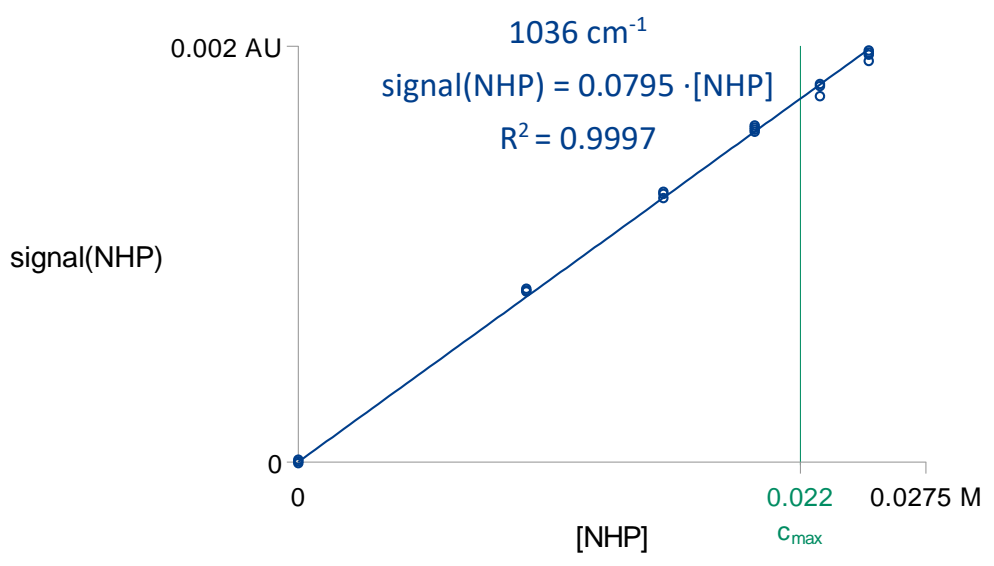

Figure S5 - The NHP signal is proportional to the concentration.

These experiments demonstrate that the observed signals are proportional to the analyte concentration over the reaction concentrations for $\mathrm{N}$-(phenylseleno)phthalimide and phthalimide. 


\section{Derivation of Equations 1 and 2}

\subsection{Standard addition of a reactant (Equation 1)}

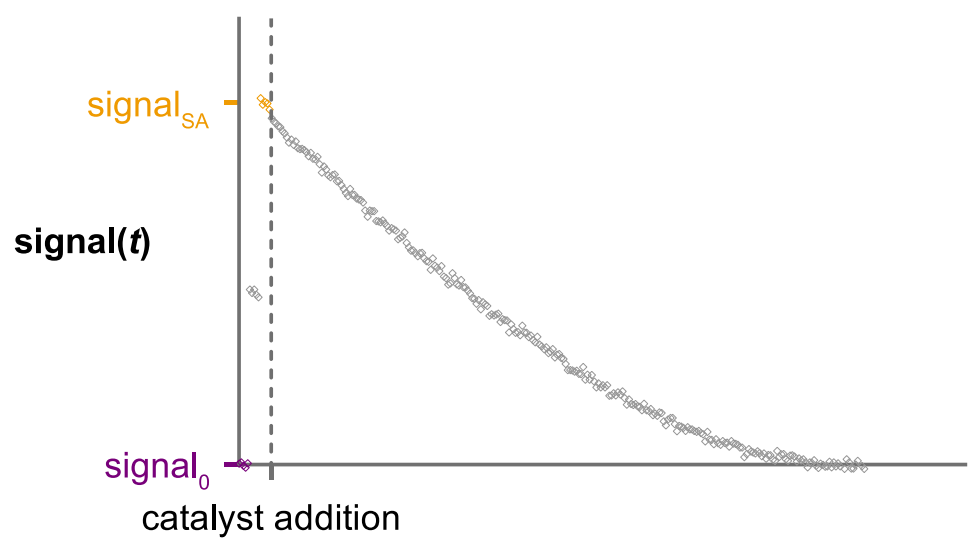

Figure S6 - FTIR data highlights the key values required for calibration using the standard addition of a reactant.

We have demonstrated that signal is proportional to concentration over the range of interest:

$$
[\mathrm{R}]_{t}=\mathbf{k}_{\mathrm{R}} \cdot \operatorname{signal}(t)
$$

The concentration before and after the standard addition of reactant $(R)$ was known. Hence, $\mathbf{k}_{R}$ can be calculated from known values:

$$
\mathbf{k}_{\mathrm{R}}=\frac{\mathrm{c}_{\mathrm{SA}}-\mathrm{c}_{0}}{\text { signal }_{\mathrm{SA}}-\operatorname{signal}_{0}}
$$

Since both signal ${ }_{0}$ and $c_{0}$ are zero, the expression for $\mathbf{k}_{\mathrm{R}}$ simplifies and the concentration of reactant can be calculated:

$$
[\mathrm{R}]_{t}=\frac{\mathrm{c}_{\mathrm{SA}}}{\operatorname{signal}_{\mathrm{SA}}} \cdot \operatorname{signal}(t)=\frac{\mathrm{n}_{\mathrm{SA}}}{\mathrm{V}_{\mathrm{SA}} \cdot \operatorname{signal}_{\mathrm{SA}}} \cdot \operatorname{signal}(t)
$$

In these formulas, signal $\left.\right|_{S A}$ is the signal height after the standard addition, signal ${ }_{0}$ is the signal height before any addition of the reactant, $\mathrm{c}_{\mathrm{SA}}$ is the concentration after addition, $\mathrm{c}_{0}$ is the concentration before addition, $\mathrm{n}_{\mathrm{SA}}$ is the amount of reactant added and $\mathrm{V}_{\mathrm{SA}}$ is the volume after the addition. 


\subsection{Standard addition of product (Equation 2)}

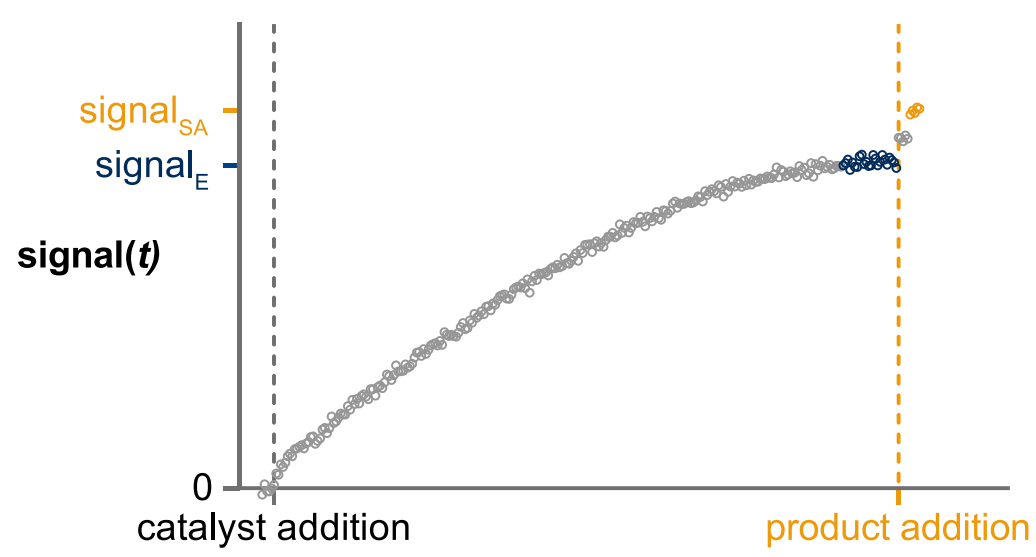

Figure S7 - FTIR data highlights the key values required for calibration using the standard addition of a product.

The calibration using the standard addition of a product calibration technique uses the fact that the amount of product $(P)$ present after the standard addition, $\left([P]_{S A} \cdot V_{S A}\right)$, must be equal to the amount present at the end of the reaction, $\left([\mathrm{P}]_{\mathrm{E}} \cdot \mathrm{V}_{\mathrm{E}}\right)$, plus the amount added in the standard addition $\left(\mathrm{n}_{\mathrm{SA}}\right)$ :

$$
\left([\mathrm{P}]_{\mathrm{SA}} \cdot \mathrm{V}_{\mathrm{SA}}\right)=\left([\mathrm{P}]_{\mathrm{E}} \cdot \mathrm{V}_{\mathrm{E}}\right)+\mathrm{n}_{\mathrm{SA}}
$$

Since signal is proportional to concentration $\left([\mathrm{P}]_{t}=\mathbf{k}_{\mathrm{P}} \cdot \operatorname{signal}(t)\right)$, substituting gives the $\mathbf{k}_{\mathrm{P}}$ in terms of known values:

$$
\mathbf{k}_{\mathrm{P}}=\frac{\mathrm{n}_{\mathrm{SA}}}{\left(\mathrm{V}_{\mathrm{SA}} \cdot \operatorname{signal}_{\mathrm{SA}}\right)-\left(\mathrm{V}_{\mathrm{E}} \cdot \operatorname{signal}_{\mathrm{E}}\right)}
$$

Therefore, the concentration of product present at each time point can be calculated:

$$
[\mathrm{P}]_{t}=\frac{\mathrm{n}_{\mathrm{SA}}}{\left(\mathrm{V}_{\mathrm{SA}} \cdot \operatorname{signal}_{\mathrm{SA}}\right)-\left(\mathrm{V}_{\mathrm{E}} \cdot \operatorname{signal}_{\mathrm{E}}\right)} \cdot \operatorname{signal}(t)
$$

In these formulas, signal ${ }_{S A}$ is the signal height after the standard addition, signal $\left.\right|_{E}$ is the signal height at the end of the reaction, $\mathrm{n}_{\mathrm{SA}}$ is the amount of product added, $\mathrm{V}_{\mathrm{E}}$ is the volume of the reaction, $\mathrm{V}_{\mathrm{SA}}$ is the volume after the standard addition, $[\mathrm{P}]_{\mathrm{E}}$ is the concentration of product at the end of the reaction and $[\mathrm{P}]_{\mathrm{SA}}$ is the concentration of product after standard addition. 


\section{Application of the standard addition method to the organocatalytic $\alpha$-selenylation of aldehydes}

\subsection{Standard reaction}

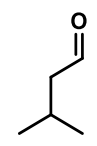

$0.103 \mathrm{M}$ 5.6 equiv

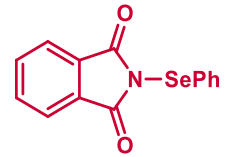

$0.0185 \mathrm{M}$

1.0 equiv

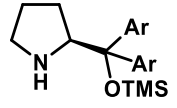

$0.6 \mathrm{~mol} \%$

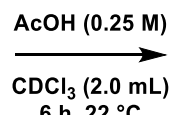

$6 \mathrm{~h}, 22{ }^{\circ} \mathrm{C}$

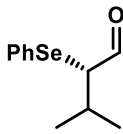<smiles>O=C1NC(=O)c2ccccc21</smiles>

$100 \%$

Stock solutions were used in the preparation of this reaction.

SL Reagent

A isovaleraldehyde

B $\mathrm{AcOH}$

C 1,1,2,2-tetrachloroethane

D $\quad \mathrm{N}$-(phenylseleno)phthalimide (98\%)

E catalyst

F phthalimide

\section{Volume / mL}

1.00

1.00

2.00

1.00

2.00

2.00
Mass / mg

35.5

60.4

34.2

22.6

2.5

10.0
Concentration / M

0.412

1.006

0.102

0.073

0.002

0.034

A vial fitted with a septum, the ReactIR probe and a stirrer bar was charged successively with stock solutions A ( $500 \mu \mathrm{L}, 0.206 \mathrm{mmol}, 5.6$ equiv), B ( $500 \mu \mathrm{L}, 0.503 \mathrm{mmol})$ and C ( $400 \mu \mathrm{L}, 0.041 \mathrm{mmol})$. Five consecutive FTIR spectra were taken before stock solution $\mathbf{D}(500 \mu \mathrm{L}, 0.037 \mathrm{mmol}, 1.0$ equiv) was added in two portions ( $200 \mu \mathrm{L}, 300 \mu \mathrm{L}$ ). Five consecutive FTIR spectra were taken after both additions before stock $\mathrm{E}\left(100 \mu \mathrm{L}, 2.1 \times 10^{-4} \mathrm{mmol}, 0.6 \mathrm{~mol} \%\right)$ was added to initiate the reaction. FTIR spectra were recorded consecutively until the reaction appeared to end by visual analysis of the data. Stock solution F (500 $\mu \mathrm{L}, 0.017 \mathrm{mmol})$ was added in two portions (200 $\mu \mathrm{L}, 300 \mu \mathrm{L})$. Immediately after the second addition of stock solution $F$, a $0.6 \mathrm{~mL}$ aliquot of the reaction was taken, frozen in liquid $\mathrm{N}_{2}$, and analyzed by qNMR. The spectroscopic data of the reduced product, 3-methyl-2-(phenylselenyl)butanol, matched that from the literature. ${ }^{1}$ The FTIR data is tabulated in Appendix 3 (S-17).

Both additions of NPSP were used to calculate the concentration of reactant. The values are tabulated below.

$$
\mathrm{C}_{S A} / \mathrm{M}
$$

calibration 1

0.0092

0.000771

11.9 calibration 2

0.0193

0.00162

11.9

Final concentration of reactant $=0.000 \mathrm{M}$ 


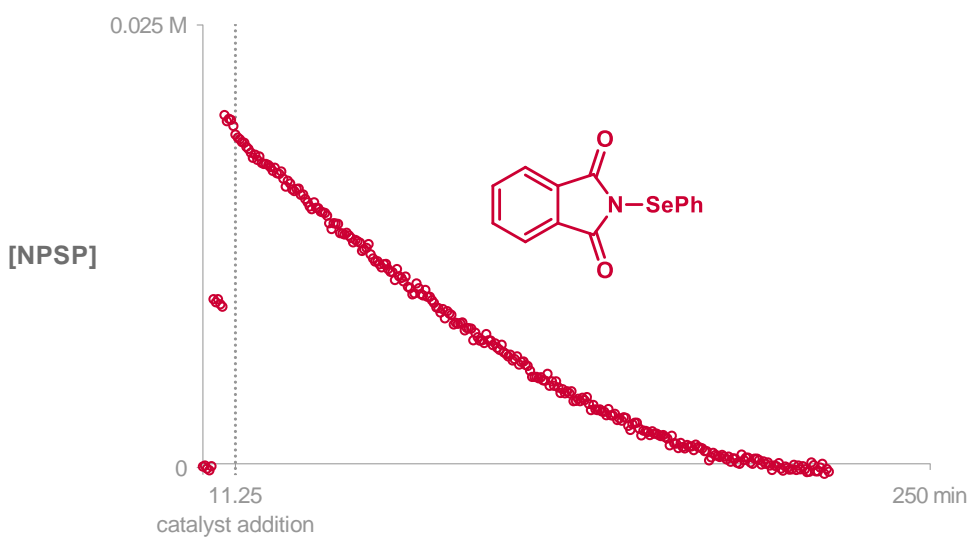

Figure S8 - The calibrated concentration of NPSP.

Both additions of NHP were used to calculate the concentration of product. The values are tabulated below.

\begin{tabular}{|c|c|c|}
\hline & calibration 1 & calibration 2 \\
\hline $\mathrm{n}_{\mathrm{SA}} / \mathrm{mmol}$ & 0.0068 & 0.0102 \\
\hline $\mathrm{V}_{\mathrm{E}} / \mathrm{mL}$ & 2.00 & 2.20 \\
\hline $\mathrm{V}_{\mathrm{SA}} / \mathrm{mL}$ & 2.20 & 2.50 \\
\hline signal $_{E}$ / A.U. & 0.001404 & 0.001509 \\
\hline signal $_{S A} /$ A.U. & 0.001509 & 0.001627 \\
\hline $\mathbf{k}_{\mathrm{P}} / \mathrm{M} / \mathrm{A} . U$. & 13.3 & 13.6 \\
\hline
\end{tabular}

Final concentration of product $=0.0189 \mathrm{M}$

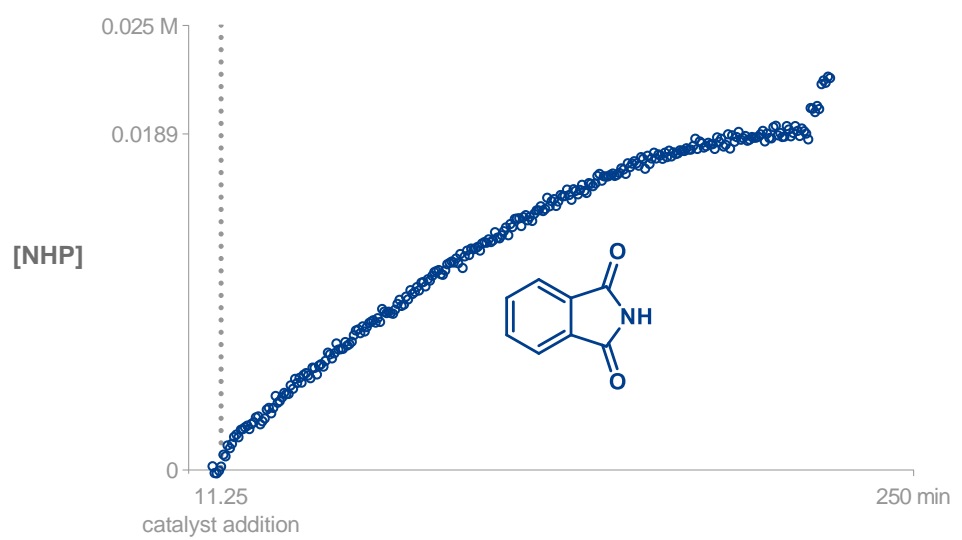

Figure S9 - The calibrated concentration of NHP.

Quantitative NMR was used to confirm the yield. We set the integral of the internal standard protons, to 200 and used the average relative integral of the product, 3-methyl-2-(phenylselenyl)butanal, to determine the NMR yield. 


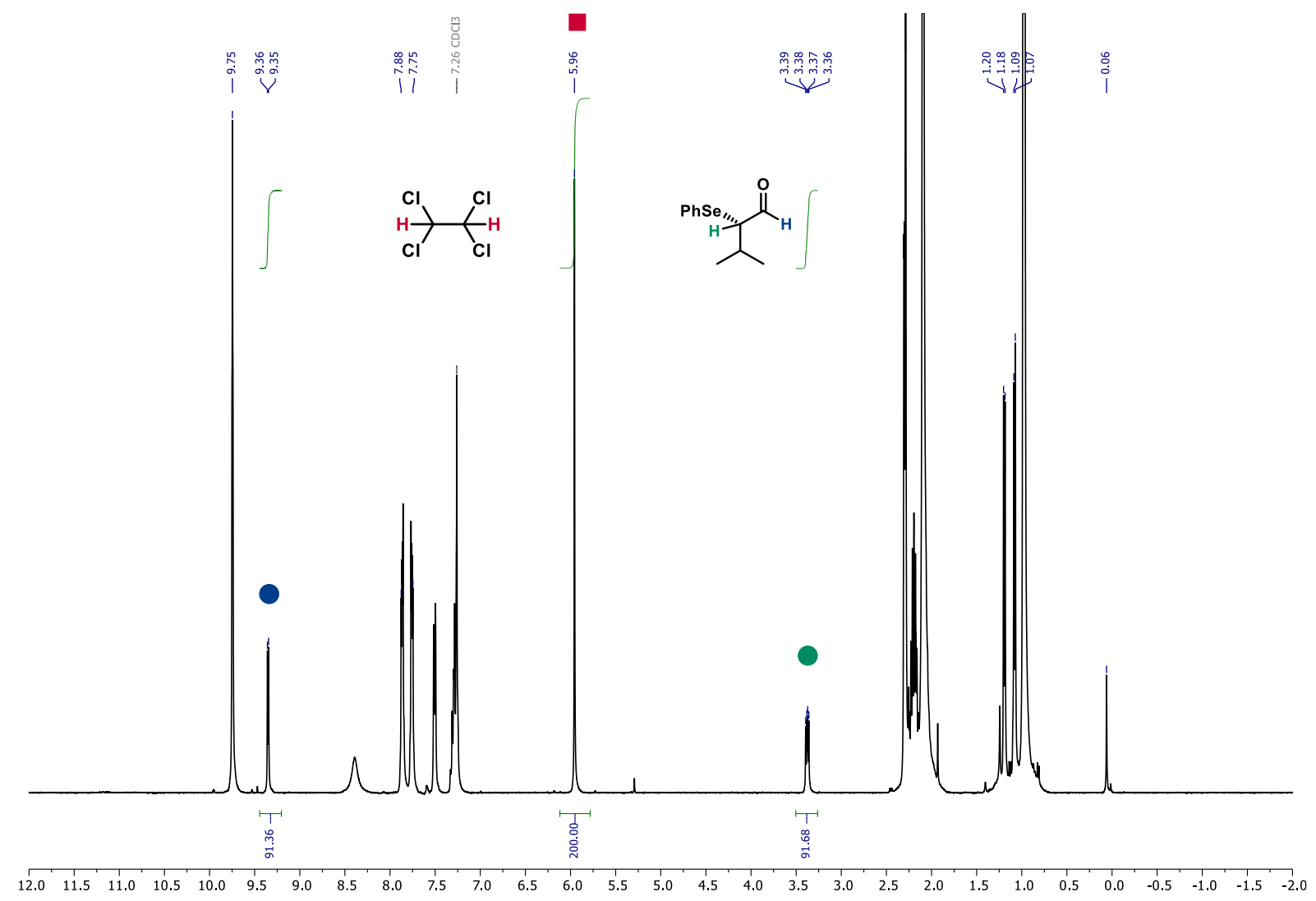

Figure $S 10$ - Quantitative $\mathrm{NMR}\left(\mathrm{CDCl}_{3}, 400 \mathrm{MHz}\right)$ used to calculate the reaction yield.

$[I S]=0.0204 \mathrm{M}$

Average integral of product $=(91.36+91.68) / 2=91.52$

[product $]=[I S]^{*} 91.52 / 100$

[product] $=0.0187 \mathrm{M}$

The final concentration of product determined by qNMR was used to calibrate the FTIR data.

Reactant, where signal is that measured for $N$-(phenylseleno)phthalimide $\left(862 \mathrm{~cm}^{-1}\right)$ :

$$
[\mathrm{R}]_{t}=\frac{(\mathrm{Y})}{\operatorname{signal}_{\mathrm{s}}-\operatorname{signal}_{\mathrm{E}}} \cdot \operatorname{signal}(t)
$$

Product, where signal is that measured for phthalimide $\left(1036 \mathrm{~cm}^{-1}\right)$ :

$$
[\mathrm{P}]_{t}=\frac{(\mathrm{Y})}{\operatorname{signal}_{\mathrm{E}}} \cdot \operatorname{signal}(t)
$$

In these formulas, $Y$ represents the NMR yield, signal $\left.\right|_{E}$ is the signal height at the end of the reaction before the standard addition of product and signal ${ }_{s}$ is the measured signal height at the start of the reaction. 


\subsection{Reaction with reduced catalyst loading}

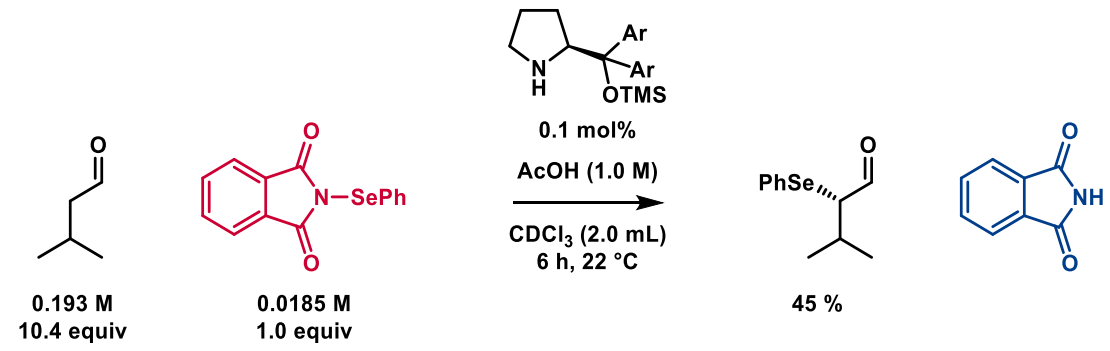

Stock solutions were used in the preparation of this reaction.

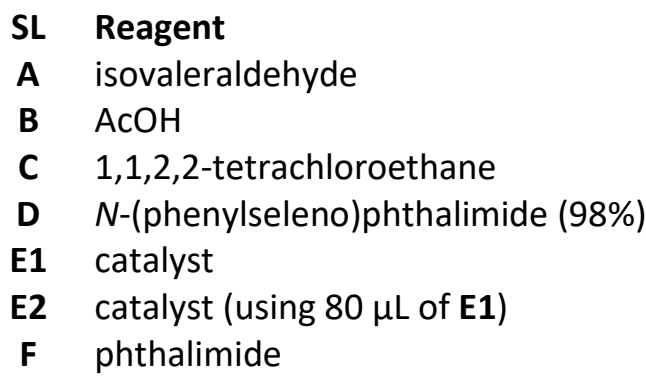

$\begin{array}{ccc}\text { Volume / } \mathbf{~ L ~} & \text { Mass / mg } & \text { Concentration / M } \\ 1.00 & 66.3 & 0.770 \\ 1.00 & 239.8 & 3.993 \\ 2.00 & 34.2 & 0.102 \\ 1.00 & 22.6 & 0.073 \\ 2.00 & 5.7 & 0.005 \\ 1.00 & 0.23 & 3.8 \times 10^{-4} \\ 2.00 & 10.0 & 0.034\end{array}$

A vial fitted with a septum, the ReactIR probe and a stirrer bar was charged successively with stock solutions A (500 $\mu \mathrm{L}, 0.385 \mathrm{mmol}, 10$ equiv), B (500 $\mu \mathrm{L}, 1.997 \mathrm{mmol})$ and C (400 $\mu \mathrm{L}, 0.041 \mathrm{mmol})$. Five consecutive FTIR spectra were taken before stock solution $\mathbf{D}(500 \mu \mathrm{L}, 0.037 \mathrm{mmol}, 1.0$ equiv) was added in two portions ( $200 \mu \mathrm{L}, 300 \mu \mathrm{L}$ ). Five consecutive FTIR spectra were taken after each addition before stock E2 (100 $\left.\mu \mathrm{L}, 3.8 \times 10^{-5} \mathrm{mmol}, 0.1 \mathrm{~mol} \%\right)$ was added to initiate the reaction. FTIR spectra were recorded consecutively until the reaction appeared to stall by visual analysis of the data. Stock solution $\mathbf{F}(500 \mu \mathrm{L}, 0.017 \mathrm{mmol})$ was added in two portions $(200 \mu \mathrm{L}, 300 \mu \mathrm{L})$. Immediately after the second addition of stock solution $\mathbf{F}$, a $0.6 \mathrm{~mL}$ aliquot of the reaction was taken, frozen in liquid $\mathrm{N}_{2}$, and analyzed by qNMR. The spectroscopic data of the reduced product, 3-methyl-2(phenylselenyl)butanol, matched that from the literature. ${ }^{1}$ The FTIR data is tabulated in Appendix 4 (S-24).

Both additions of NPSP were used to calculate the concentration of reactant. The values used in the calibration are tabulated below.

$$
\begin{aligned}
& \mathrm{C}_{S A} / \mathrm{M} \\
& \text { signal }_{S A} / \text { A.U. } \\
& \mathbf{k}_{\mathrm{R}} / \mathrm{M} \text { /A.U. }
\end{aligned}
$$

calibration 1
0.0092
0.000668
13.8

13.8

\author{
calibration 2 \\ 0.0193 \\ 0.00147
}

13.1

Final concentration of reactant $=0.0102 \mathrm{M}$ 


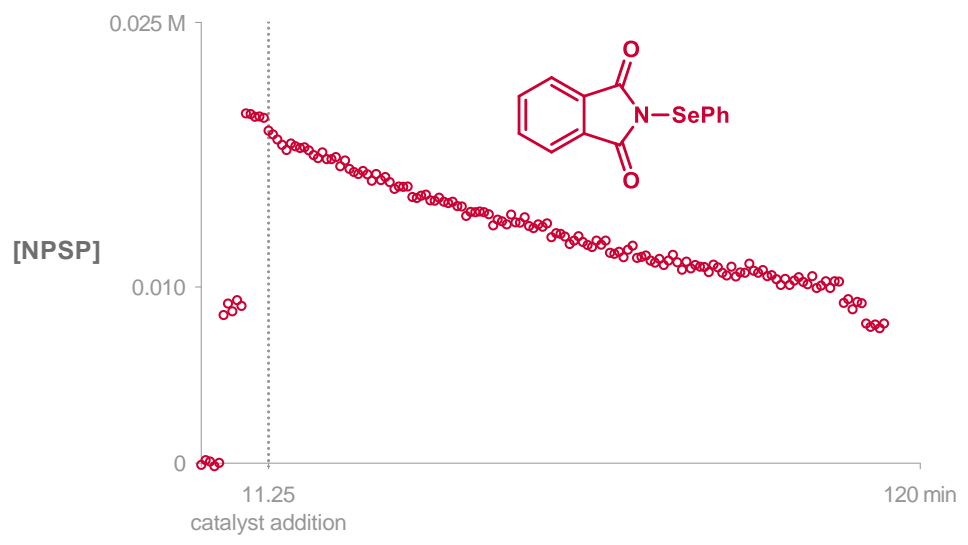

Figure S11 - The calibrated concentration of NPSP.

Both additions of NHP were used to calculate the concentration of product. The values are tabulated below. We used a baseline at $1060 \mathrm{~cm}^{-1}$, rather than $1058 \mathrm{~cm}^{-1}$, because of the increased AcOH signal $\left(1052 \mathrm{~cm}^{-1}\right)$.

$\begin{array}{lcc} & \text { calibration } 1 & \text { calibration } 2 \\ \mathrm{n}_{\mathrm{SA}} / \mathrm{mmol} & 0.0068 & 0.0102 \\ \mathrm{~V}_{\mathrm{E}} / \mathrm{mL} & 2.00 & 2.20 \\ \mathrm{~V}_{\mathrm{SA}} / \mathrm{mL} & 2.20 & 2.50 \\ \text { signal }_{\mathrm{E}} / \mathrm{A} . U . & 0.000645 & 0.000821 \\ \text { signal } & 0.000821 & 0.001030 \\ \mathrm{k}_{\mathrm{P}} / \mathrm{M} / \mathrm{A} \text { A.U. } & 13.2 & 13.3\end{array}$

Final concentration of product $=0.0085 \mathrm{M}$

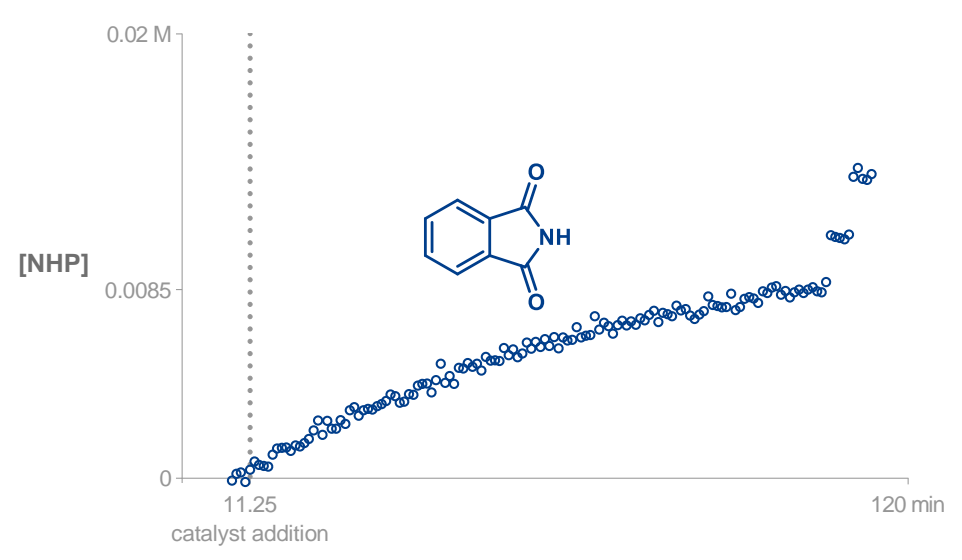

Figure S12 - The calibrated concentration of NHP.

Quantitative NMR was used to confirm the yield. The integral of the internal standard protons, highlighted, was set to 200 and the average relative integral of the product, 3-methyl-2(phenylselenyl)butanal, was then used to determine the NMR yield. 


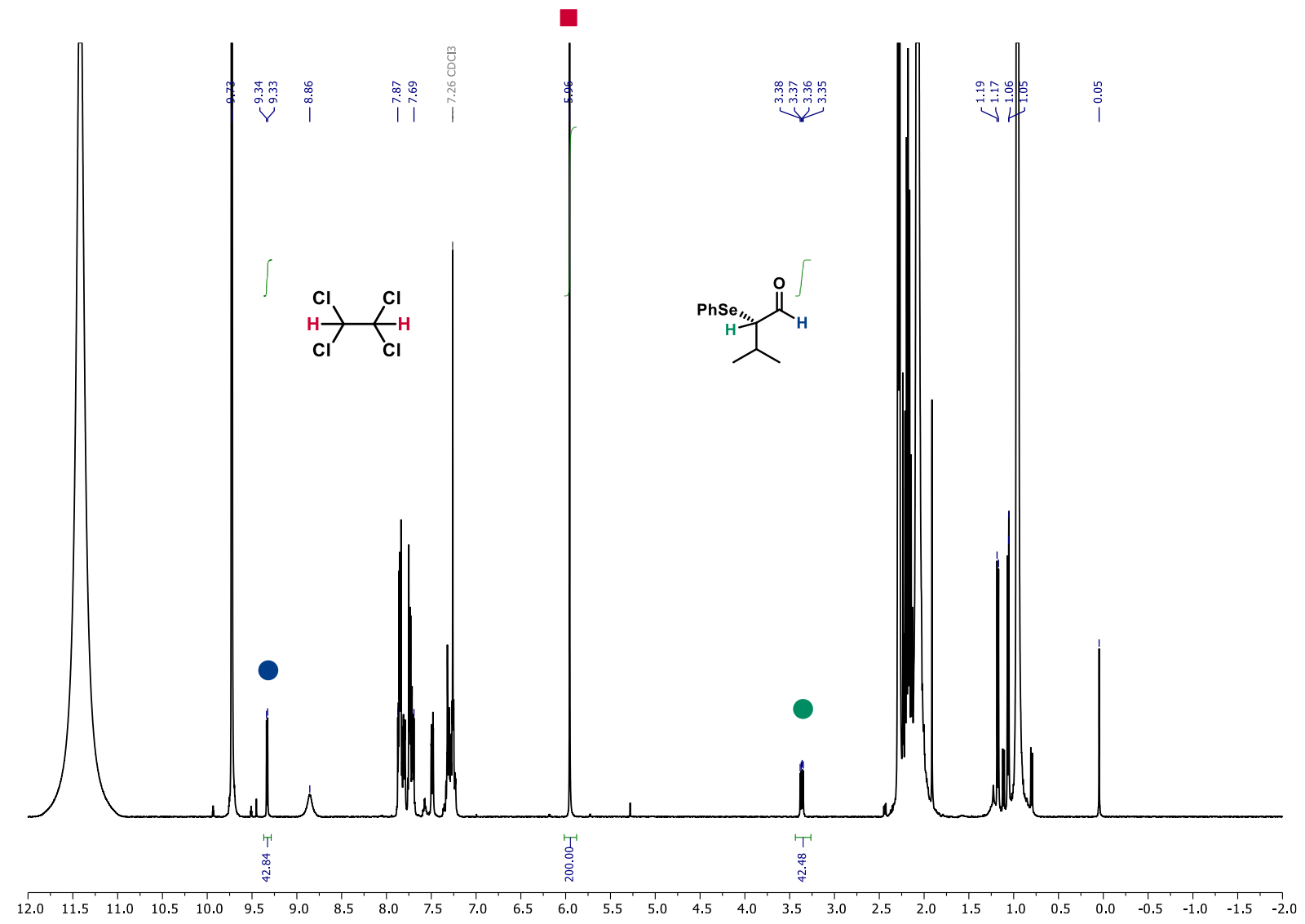

Figure $S 13$ - Quantitative $N M R\left(C C_{3}, 400 \mathrm{MHz}\right)$ used to calculate the reaction yield.

$[I S]=0.0204 \mathrm{M}$

Average integral of product $=(42.84+42.48) / 2=42.66$

[product $]=[\text { IS }]^{*} 42.66 / 100$

[product] $=0.0087 \mathrm{M}$

The final concentration of product determined by qNMR was used to calibrate the FTIR data.

Reactant, where signal is that measured for $N$-(phenylseleno)phthalimide $\left(862 \mathrm{~cm}^{-1}\right)$ :

$$
[\mathrm{R}]_{t}=\frac{(\mathrm{Y})}{\operatorname{signal}_{\mathrm{S}}-\operatorname{signal}_{\mathrm{E}}} \cdot \operatorname{signal}(t)
$$

Product, where signal is that measured for phthalimide $\left(1036 \mathrm{~cm}^{-1}\right)$ :

$$
[\mathrm{P}]_{t}=\frac{(\mathrm{Y})}{\operatorname{signal}_{\mathrm{E}}} \cdot \operatorname{signal}(t)
$$

In these formulas, $Y$ represents the NMR yield, signal $\left.\right|_{E}$ is the signal height at the end of the reaction before the standard addition of product and signals is the measured signal height at the start of the reaction. 


\section{Requirements of the standard addition method}

The standard addition calibration requires that the reactants or products used in the standard addition are of known purity. As for any other spectroscopic technique, it is essential that the FTIR peaks used for the analysis are well resolved and accurately reflect the concentration of the reactant or product of interest.

\section{References}

1 - Tiecco, M.; Carlone, A.; Sternativo, S.; Marini, F.; Bartoli, G.; Melchiorre, P. Organocatalytic Asymmetric $\alpha$-Selenenylation of Aldehydes, Angew. Chem., Int. Ed., 2007, 46, 6882-6885. 
8. Appendix 1 - Data for figures $\mathrm{S2}$ and $\mathrm{S} 3$

\begin{tabular}{|c|c|c|}
\hline time / min & {$[N P S P] / M$} & peak at $862 \mathrm{~cm}^{-1}$ \\
\hline 0 & 0 & -0.000014 \\
\hline 0.75 & 0 & 0.000002 \\
\hline 1.5 & 0 & 0.000016 \\
\hline 2.25 & 0 & -0.000003 \\
\hline 3 & 0 & 0.000000 \\
\hline 3.75 & 0.00875 & 0.000645 \\
\hline 4.5 & 0.00875 & 0.000641 \\
\hline 5.25 & 0.00875 & 0.000643 \\
\hline 6 & 0.00875 & 0.000617 \\
\hline 6.75 & 0.00875 & 0.000643 \\
\hline 7.5 & 0.014 & 0.001032 \\
\hline 8.25 & 0.014 & 0.001017 \\
\hline 9 & 0.014 & 0.000999 \\
\hline 9.75 & 0.014 & 0.001012 \\
\hline 10.5 & 0.014 & 0.001005 \\
\hline 11.25 & 0.0175 & 0.001243 \\
\hline 12 & 0.0175 & 0.001235 \\
\hline 12.75 & 0.0175 & 0.001253 \\
\hline 13.5 & 0.0175 & 0.001241 \\
\hline 14.25 & 0.0175 & 0.001235 \\
\hline 15 & 0.02 & 0.001439 \\
\hline 15.75 & 0.02 & 0.001429 \\
\hline 16.5 & 0.02 & 0.001422 \\
\hline 17.25 & 0.02 & 0.001409 \\
\hline 18 & 0.02 & 0.001416 \\
\hline 18.75 & 0.021875 & 0.001532 \\
\hline 19.5 & 0.021875 & 0.001515 \\
\hline 20.25 & 0.021875 & 0.001539 \\
\hline 21 & 0.021875 & 0.001541 \\
\hline 21.75 & 0.021875 & 0.001534 \\
\hline
\end{tabular}


9. Appendix 2-Data for figures $\mathrm{S} 5$ and $\mathrm{S} 6$

\begin{tabular}{|c|c|c|}
\hline time / $\min$ & {$[\mathrm{NHP}] / \mathrm{M}$} & peak at $1036 \mathrm{~cm}^{-1}$ \\
\hline 0 & 0 & $3.05 E-06$ \\
\hline 0.75 & 0 & $1.11 \mathrm{E}-05$ \\
\hline 1.5 & 0 & $1.77 \mathrm{E}-06$ \\
\hline 2.25 & 0 & $-6.2 E-06$ \\
\hline 3 & 0 & $-9.7 E-06$ \\
\hline 3.75 & 0.01 & 0.000833 \\
\hline 4.5 & 0.01 & 0.000833 \\
\hline 5.25 & 0.01 & 0.000825 \\
\hline 6 & 0.01 & 0.000835 \\
\hline 6.75 & 0.01 & 0.00082 \\
\hline 7.5 & 0.016 & 0.001272 \\
\hline 8.25 & 0.016 & 0.001302 \\
\hline 9 & 0.016 & 0.001296 \\
\hline 9.75 & 0.016 & 0.00129 \\
\hline 10.5 & 0.016 & 0.001298 \\
\hline 11.25 & 0.02 & 0.001594 \\
\hline 12 & 0.02 & 0.001605 \\
\hline 12.75 & 0.02 & 0.001598 \\
\hline 13.5 & 0.02 & 0.001612 \\
\hline 14.25 & 0.02 & 0.001619 \\
\hline 15 & 0.022857 & 0.001763 \\
\hline 15.75 & 0.022857 & 0.00181 \\
\hline 16.5 & 0.022857 & 0.001805 \\
\hline 17.25 & 0.022857 & 0.001813 \\
\hline 18 & 0.022857 & 0.001813 \\
\hline 18.75 & 0.022857 & 0.001815 \\
\hline 19.5 & 0.025 & 0.001933 \\
\hline 20.25 & 0.025 & 0.001967 \\
\hline 21 & 0.025 & 0.00196 \\
\hline 21.75 & 0.025 & 0.001968 \\
\hline 22.5 & 0.025 & 0.001978 \\
\hline
\end{tabular}


10. Appendix 3 - Data for figures 1, 2, 3, 4, S6, S8 and S9

\begin{tabular}{|c|c|c|c|c|}
\hline time / min & $\begin{array}{c}\text { raw peak at } \\
862 \mathrm{~cm}^{-1}\end{array}$ & $\begin{array}{l}\text { normalised peak } \\
\text { at } 862 \mathrm{~cm}^{-1}\end{array}$ & $\begin{array}{c}\text { raw peak at } \\
1036 \mathrm{~cm}^{-1}\end{array}$ & $\begin{array}{c}\text { normalised peak } \\
\text { at } 1036 \mathrm{~cm}^{-1}\end{array}$ \\
\hline 0.00 & -0.00138 & $4.38 \mathrm{E}-06$ & -0.0003 & 0.000222 \\
\hline 0.75 & -0.00134 & $9.13 \mathrm{E}-06$ & -0.0003 & 0.000191 \\
\hline 1.50 & -0.00136 & $-5.7 \mathrm{E}-06$ & -0.0003 & 0.000242 \\
\hline 2.25 & -0.00137 & $-1.4 \mathrm{E}-05$ & -0.00031 & 0.000201 \\
\hline 3.00 & -0.00136 & $5.88 \mathrm{E}-06$ & -0.00031 & 0.000204 \\
\hline 3.75 & -0.00062 & 0.000786 & -0.00027 & 0.000101 \\
\hline 4.50 & -0.00061 & 0.000771 & -0.00026 & 9.87E-05 \\
\hline 5.25 & -0.00063 & 0.000785 & -0.00027 & 0.000119 \\
\hline 6.00 & -0.00064 & 0.000761 & -0.00026 & 0.00012 \\
\hline 6.75 & -0.00064 & 0.000751 & -0.00025 & 0.00012 \\
\hline 7.50 & 0.000186 & 0.001644 & -0.00023 & $-2.8 \mathrm{E}-05$ \\
\hline 8.25 & 0.000157 & 0.001615 & -0.00021 & $1.62 \mathrm{E}-05$ \\
\hline 9.00 & 0.000155 & 0.001626 & -0.00021 & $-1.4 \mathrm{E}-05$ \\
\hline 9.75 & 0.000157 & 0.001621 & -0.00021 & $-1.4 \mathrm{E}-05$ \\
\hline 10.50 & 0.000157 & 0.001592 & -0.00021 & $-2.1 E-06$ \\
\hline 11.25 & $7.92 \mathrm{E}-05$ & 0.001553 & -0.00021 & $1.4 \mathrm{E}-05$ \\
\hline 12.00 & $5.60 \mathrm{E}-05$ & 0.001538 & -0.00019 & $6.36 \mathrm{E}-05$ \\
\hline 12.75 & $3.57 E-05$ & 0.001531 & -0.00019 & $5.75 \mathrm{E}-05$ \\
\hline 13.50 & $1.01 \mathrm{E}-05$ & 0.001518 & -0.00017 & 0.000102 \\
\hline 14.25 & $9.51 E-06$ & 0.001514 & -0.00018 & $9.12 \mathrm{E}-05$ \\
\hline 15.00 & $3.55 \mathrm{E}-05$ & 0.001495 & -0.00016 & 0.00011 \\
\hline 15.75 & $8.82 \mathrm{E}-06$ & 0.001486 & -0.00016 & 0.000138 \\
\hline 16.50 & $-1.73 E-05$ & 0.001468 & -0.00015 & 0.000149 \\
\hline 17.25 & $-4.83 E-05$ & 0.001444 & -0.00015 & 0.000137 \\
\hline 18.00 & $-1.61 E-05$ & 0.001458 & -0.00013 & 0.000168 \\
\hline 18.75 & $-2.74 \mathrm{E}-05$ & 0.001434 & -0.00013 & 0.000171 \\
\hline 19.50 & $-4.19 E-05$ & 0.00145 & -0.00014 & 0.000178 \\
\hline 20.25 & $-7.63 E-05$ & 0.001419 & -0.00013 & 0.000185 \\
\hline 21.00 & $-2.83 E-05$ & 0.001415 & -0.00012 & 0.000171 \\
\hline 21.75 & $-6.73 E-05$ & 0.001416 & -0.00011 & 0.000193 \\
\hline 22.50 & $-7.22 \mathrm{E}-05$ & 0.001411 & -0.00012 & 0.0002 \\
\hline 23.25 & $-9.70 E-05$ & 0.001401 & $-9.58 E-05$ & 0.00022 \\
\hline 24.00 & $-8.28 \mathrm{E}-05$ & 0.001384 & -0.00011 & 0.000223 \\
\hline 24.75 & $-6.22 \mathrm{E}-05$ & 0.001399 & -0.0001 & 0.000191 \\
\hline 25.50 & -0.0001 & 0.001372 & $-9.64 \mathrm{E}-05$ & 0.000204 \\
\hline 26.25 & $-8.87 E-05$ & 0.001369 & $-8.67 \mathrm{E}-05$ & 0.000215 \\
\hline 27.00 & -0.00011 & 0.001381 & $-7.70 \mathrm{E}-05$ & 0.000253 \\
\hline 27.75 & -0.00012 & 0.001347 & -7.97E-05 & 0.00026 \\
\hline 28.50 & -0.00016 & 0.00131 & $-7.26 \mathrm{E}-05$ & 0.000237 \\
\hline 29.25 & -0.00015 & 0.001337 & $-6.09 E-05$ & 0.000259 \\
\hline 30.00 & -0.00014 & 0.001324 & $-5.19 E-05$ & 0.000309 \\
\hline 30.75 & -0.0002 & 0.001297 & $-3.88 \mathrm{E}-05$ & 0.000281 \\
\hline 31.50 & -0.00018 & 0.001289 & $-5.36 \mathrm{E}-05$ & 0.000288 \\
\hline 32.25 & -0.00015 & 0.001299 & $-4.63 E-05$ & 0.000303 \\
\hline 33.00 & -0.00021 & 0.001301 & $-4.70 \mathrm{E}-05$ & 0.000322 \\
\hline
\end{tabular}




\begin{tabular}{|c|c|c|c|c|}
\hline 33.75 & -0.00019 & 0.001272 & $-3.81 E-05$ & 0.000317 \\
\hline 34.50 & -0.00021 & 0.001273 & $-2.48 \mathrm{E}-05$ & 0.000318 \\
\hline 35.25 & -0.00022 & 0.001253 & $-1.65 E-05$ & 0.000354 \\
\hline 36.00 & -0.00023 & 0.001237 & $-1.76 \mathrm{E}-05$ & 0.000339 \\
\hline 36.75 & -0.00023 & 0.001218 & $-5.75 E-07$ & 0.000381 \\
\hline 37.50 & -0.00025 & 0.001204 & $-9.92 E-06$ & 0.000363 \\
\hline 38.25 & -0.00023 & 0.001236 & $2.47 \mathrm{E}-06$ & 0.000385 \\
\hline 39.00 & -0.00025 & 0.001211 & $3.21 \mathrm{E}-06$ & 0.000365 \\
\hline 39.75 & -0.00026 & 0.00121 & 7.07E-07 & 0.000397 \\
\hline 40.50 & -0.00029 & 0.001193 & $2.42 \mathrm{E}-05$ & 0.000405 \\
\hline 41.25 & -0.00027 & 0.00119 & $1.92 \mathrm{E}-05$ & 0.000395 \\
\hline 42.00 & -0.0003 & 0.001186 & $1.31 \mathrm{E}-05$ & 0.000384 \\
\hline 42.75 & -0.00029 & 0.001175 & $3.33 \mathrm{E}-05$ & 0.000427 \\
\hline 43.50 & -0.00032 & 0.001139 & $2.86 \mathrm{E}-05$ & 0.000427 \\
\hline 44.25 & -0.00033 & 0.001112 & $3.58 \mathrm{E}-05$ & 0.000399 \\
\hline 45.00 & -0.00032 & 0.001139 & $3.74 \mathrm{E}-05$ & 0.000436 \\
\hline 45.75 & -0.00034 & 0.001137 & 4.94E-05 & 0.000442 \\
\hline 46.50 & -0.00032 & 0.001136 & $4.42 \mathrm{E}-05$ & 0.000431 \\
\hline 47.25 & -0.00036 & 0.001092 & $4.53 \mathrm{E}-05$ & 0.000456 \\
\hline 48.00 & -0.00035 & 0.001091 & $6.08 \mathrm{E}-05$ & 0.00049 \\
\hline 48.75 & -0.00037 & 0.001087 & $6.93 \mathrm{E}-05$ & 0.000483 \\
\hline 49.50 & -0.00035 & 0.001095 & $7.06 \mathrm{E}-05$ & 0.000467 \\
\hline 50.25 & -0.00038 & 0.001081 & $7.42 \mathrm{E}-05$ & 0.000488 \\
\hline 51.00 & -0.00039 & 0.001071 & $9.28 \mathrm{E}-05$ & 0.000529 \\
\hline 51.75 & -0.00041 & 0.00105 & $8.49 \mathrm{E}-05$ & 0.000503 \\
\hline 52.50 & -0.0004 & 0.001061 & 9.17E-05 & 0.000504 \\
\hline 53.25 & -0.00044 & 0.001055 & $8.86 \mathrm{E}-05$ & 0.000506 \\
\hline 54.00 & -0.00041 & 0.001049 & $9.71 \mathrm{E}-05$ & 0.000534 \\
\hline 54.75 & -0.00044 & 0.001011 & $9.81 \mathrm{E}-05$ & 0.00052 \\
\hline 55.50 & -0.00043 & 0.001017 & $9.71 \mathrm{E}-05$ & 0.000526 \\
\hline 56.25 & -0.00044 & 0.001024 & 0.000121 & 0.000536 \\
\hline 57.00 & -0.00042 & 0.001042 & 0.000112 & 0.000563 \\
\hline 57.75 & -0.00047 & 0.000994 & 0.000125 & 0.000584 \\
\hline 58.50 & -0.00047 & 0.000975 & 0.000122 & 0.000587 \\
\hline 59.25 & -0.00048 & 0.00096 & 0.000135 & 0.000571 \\
\hline 60.00 & -0.00049 & 0.000961 & 0.000144 & 0.0006 \\
\hline 60.75 & -0.00052 & 0.000948 & 0.000136 & 0.000579 \\
\hline 61.50 & -0.00051 & 0.000934 & 0.000128 & 0.000597 \\
\hline 62.25 & -0.0005 & 0.000948 & 0.000147 & 0.000615 \\
\hline 63.00 & -0.00049 & 0.000949 & 0.000153 & 0.000621 \\
\hline 63.75 & -0.00049 & 0.000925 & 0.000169 & 0.000625 \\
\hline 64.50 & -0.00052 & 0.000912 & 0.000164 & 0.000616 \\
\hline 65.25 & -0.00055 & 0.00091 & 0.000166 & 0.000635 \\
\hline 66.00 & -0.00056 & 0.000874 & 0.00016 & 0.000618 \\
\hline 66.75 & -0.00053 & 0.000926 & 0.000187 & 0.000673 \\
\hline 67.50 & -0.00056 & 0.000892 & 0.000189 & 0.000661 \\
\hline 68.25 & -0.00054 & 0.000883 & 0.000177 & 0.000656 \\
\hline 69.00 & -0.00057 & 0.000868 & 0.000179 & 0.000662 \\
\hline
\end{tabular}




\begin{tabular}{|c|c|c|c|c|}
\hline 69.75 & -0.00057 & 0.00089 & 0.000196 & 0.000656 \\
\hline 70.50 & -0.0006 & 0.000843 & 0.00018 & 0.000653 \\
\hline 71.25 & -0.00063 & 0.000837 & 0.000182 & 0.00067 \\
\hline 72.00 & -0.00064 & 0.000807 & 0.000202 & 0.000695 \\
\hline 72.75 & -0.00063 & 0.000813 & 0.000217 & 0.000712 \\
\hline 73.50 & -0.00063 & 0.000858 & 0.00021 & 0.000686 \\
\hline 74.25 & -0.00061 & 0.000837 & 0.000219 & 0.000691 \\
\hline 75.00 & -0.00063 & 0.000808 & 0.000226 & 0.000723 \\
\hline 75.75 & -0.00062 & 0.000803 & 0.000229 & 0.000713 \\
\hline 76.50 & -0.00061 & 0.000829 & 0.000227 & 0.000751 \\
\hline 77.25 & -0.00065 & 0.000796 & 0.00024 & 0.000735 \\
\hline 78.00 & -0.00066 & 0.000795 & 0.00023 & 0.000744 \\
\hline 78.75 & -0.00067 & 0.00078 & 0.000247 & 0.000763 \\
\hline 79.50 & -0.00067 & 0.000767 & 0.00024 & 0.00075 \\
\hline 80.25 & -0.0007 & 0.000746 & 0.000263 & 0.000783 \\
\hline 81.00 & -0.0007 & 0.000743 & 0.000255 & 0.000785 \\
\hline 81.75 & -0.00072 & 0.000722 & 0.000265 & 0.000767 \\
\hline 82.50 & -0.00072 & 0.000738 & 0.000263 & 0.000797 \\
\hline 83.25 & -0.00073 & 0.000696 & 0.000269 & 0.000791 \\
\hline 84.00 & -0.00072 & 0.000729 & 0.000272 & 0.00081 \\
\hline 84.75 & -0.00071 & 0.000718 & 0.000282 & 0.000827 \\
\hline 85.50 & -0.00075 & 0.000712 & 0.000284 & 0.000831 \\
\hline 86.25 & -0.00073 & 0.000667 & 0.000294 & 0.000836 \\
\hline 87.00 & -0.00075 & 0.000675 & 0.000297 & 0.000818 \\
\hline 87.75 & -0.00076 & 0.000669 & 0.000281 & 0.000816 \\
\hline 88.50 & -0.00077 & 0.000673 & 0.000296 & 0.000834 \\
\hline 89.25 & -0.00078 & 0.000677 & 0.000304 & 0.000859 \\
\hline 90.00 & -0.00077 & 0.000639 & 0.000307 & 0.000865 \\
\hline 90.75 & -0.00077 & 0.000652 & 0.000307 & 0.00087 \\
\hline 91.50 & -0.00079 & 0.000648 & 0.000309 & 0.000866 \\
\hline 92.25 & -0.0008 & 0.000647 & 0.000311 & 0.000884 \\
\hline 93.00 & -0.00081 & 0.000593 & 0.000317 & 0.000863 \\
\hline 93.75 & -0.00082 & 0.000628 & 0.000325 & 0.000901 \\
\hline 94.50 & -0.00081 & 0.000608 & 0.000312 & 0.000843 \\
\hline 95.25 & -0.00083 & 0.000591 & 0.000338 & 0.000892 \\
\hline 96.00 & -0.00084 & 0.000594 & 0.000336 & 0.000916 \\
\hline 96.75 & -0.00085 & 0.00058 & 0.000344 & 0.000898 \\
\hline 97.50 & -0.00082 & 0.000623 & 0.00035 & 0.000926 \\
\hline 98.25 & -0.00084 & 0.000593 & 0.000336 & 0.000923 \\
\hline 99.00 & -0.00085 & 0.000592 & 0.000352 & 0.000927 \\
\hline 99.75 & -0.00085 & 0.000572 & 0.00036 & 0.000932 \\
\hline 100.50 & -0.00084 & 0.000579 & 0.000352 & 0.000917 \\
\hline 101.25 & -0.00088 & 0.000558 & 0.00036 & 0.000946 \\
\hline 102.00 & -0.0009 & 0.000548 & 0.000358 & 0.000952 \\
\hline 102.75 & -0.00088 & 0.000573 & 0.000365 & 0.000949 \\
\hline 103.50 & -0.00089 & 0.000538 & 0.000368 & 0.000964 \\
\hline 104.25 & -0.0009 & 0.00053 & 0.000381 & 0.000952 \\
\hline 105.00 & -0.0009 & 0.000518 & 0.000379 & 0.000958 \\
\hline
\end{tabular}




\begin{tabular}{|c|c|c|c|c|}
\hline 105.75 & -0.00088 & 0.000526 & 0.000387 & 0.000993 \\
\hline 106.50 & -0.00093 & 0.000499 & 0.000384 & 0.000971 \\
\hline 107.25 & -0.00094 & 0.000505 & 0.00038 & 0.000968 \\
\hline 108.00 & -0.00092 & 0.000519 & 0.0004 & 0.00098 \\
\hline 108.75 & -0.00095 & 0.00048 & 0.000409 & 0.000997 \\
\hline 109.50 & -0.00093 & 0.000492 & 0.000394 & 0.001013 \\
\hline 110.25 & -0.00094 & 0.000495 & 0.000397 & 0.000981 \\
\hline 111.00 & -0.00092 & 0.000478 & 0.000415 & 0.001027 \\
\hline 111.75 & -0.00094 & 0.000474 & 0.000407 & 0.001015 \\
\hline 112.50 & -0.00099 & 0.000452 & 0.000411 & 0.001049 \\
\hline 113.25 & -0.00099 & 0.000423 & 0.000423 & 0.001051 \\
\hline 114.00 & -0.00097 & 0.000423 & 0.000411 & 0.001052 \\
\hline 114.75 & -0.001 & 0.000423 & 0.000429 & 0.001019 \\
\hline 115.50 & -0.00098 & 0.000417 & 0.000431 & 0.001056 \\
\hline 116.25 & -0.00099 & 0.000421 & 0.000425 & 0.001065 \\
\hline 117.00 & -0.00101 & 0.000407 & 0.000434 & 0.001042 \\
\hline 117.75 & -0.00102 & 0.000405 & 0.000448 & 0.001058 \\
\hline 118.50 & -0.00099 & 0.000437 & 0.000436 & 0.001041 \\
\hline 119.25 & -0.00103 & 0.000381 & 0.000441 & 0.00107 \\
\hline 120.00 & -0.00101 & 0.000403 & 0.000443 & 0.001084 \\
\hline 120.75 & -0.00102 & 0.000381 & 0.000452 & 0.001084 \\
\hline 121.50 & -0.00103 & 0.000401 & 0.000473 & 0.001102 \\
\hline 122.25 & -0.00103 & 0.000372 & 0.00046 & 0.001082 \\
\hline 123.00 & -0.00105 & 0.000347 & 0.000458 & 0.00109 \\
\hline 123.75 & -0.00105 & 0.000365 & 0.000472 & 0.001138 \\
\hline 124.50 & -0.00106 & 0.000346 & 0.00047 & 0.001101 \\
\hline 125.25 & -0.00105 & 0.000356 & 0.00048 & 0.001122 \\
\hline 126.00 & -0.00109 & 0.000346 & 0.000465 & 0.001132 \\
\hline 126.75 & -0.00106 & 0.000356 & 0.000475 & 0.001103 \\
\hline 127.50 & -0.0011 & 0.000309 & 0.000488 & 0.001122 \\
\hline 128.25 & -0.00109 & 0.000311 & 0.000483 & 0.001148 \\
\hline 129.00 & -0.0011 & 0.00032 & 0.000498 & 0.001142 \\
\hline 129.75 & -0.00109 & 0.00031 & 0.000495 & 0.001145 \\
\hline 130.50 & -0.00109 & 0.000325 & 0.000502 & 0.001171 \\
\hline 131.25 & -0.00111 & 0.000314 & 0.000498 & 0.001149 \\
\hline 132.00 & -0.0011 & 0.000328 & 0.000492 & 0.001129 \\
\hline 132.75 & -0.00111 & 0.000297 & 0.000505 & 0.001172 \\
\hline 133.50 & -0.00112 & 0.000268 & 0.000491 & 0.001165 \\
\hline 134.25 & -0.00114 & 0.000292 & 0.000504 & 0.001151 \\
\hline 135.00 & -0.00114 & 0.000272 & 0.000507 & 0.00117 \\
\hline 135.75 & -0.00113 & 0.000267 & 0.000516 & 0.001191 \\
\hline 136.50 & -0.00113 & 0.000271 & 0.000512 & 0.001165 \\
\hline 137.25 & -0.00115 & 0.000262 & 0.000511 & 0.001157 \\
\hline 138.00 & -0.00115 & 0.000259 & 0.000525 & 0.001199 \\
\hline 138.75 & -0.00114 & 0.000244 & 0.000523 & 0.001195 \\
\hline 139.50 & -0.00115 & 0.000273 & 0.000534 & 0.001185 \\
\hline 140.25 & -0.00116 & 0.000243 & 0.000533 & 0.001197 \\
\hline 141.00 & -0.00115 & 0.000242 & 0.000524 & 0.001228 \\
\hline
\end{tabular}




\begin{tabular}{|c|c|c|c|c|}
\hline 141.75 & -0.00118 & 0.00025 & 0.000542 & 0.001237 \\
\hline 142.50 & -0.00118 & 0.000221 & 0.000545 & 0.001209 \\
\hline 143.25 & -0.0012 & 0.000229 & 0.000543 & 0.001228 \\
\hline 144.00 & -0.0012 & 0.000219 & 0.000556 & 0.001225 \\
\hline 144.75 & -0.00119 & 0.000234 & 0.000548 & 0.001227 \\
\hline 145.50 & -0.00117 & 0.000232 & 0.000546 & 0.001233 \\
\hline 146.25 & -0.00123 & 0.000195 & 0.000539 & 0.001238 \\
\hline 147.00 & -0.00122 & 0.000176 & 0.000543 & 0.001229 \\
\hline 147.75 & -0.00119 & 0.000203 & 0.000543 & 0.001232 \\
\hline 148.50 & -0.00121 & 0.000209 & 0.00056 & 0.001241 \\
\hline 149.25 & -0.00121 & 0.000209 & 0.000557 & 0.001259 \\
\hline 150.00 & -0.00122 & 0.000182 & 0.000553 & 0.001256 \\
\hline 150.75 & -0.00123 & 0.00015 & 0.00056 & 0.001237 \\
\hline 151.50 & -0.00123 & 0.000173 & 0.000557 & 0.001281 \\
\hline 152.25 & -0.00124 & 0.000171 & 0.000565 & 0.001265 \\
\hline 153.00 & -0.00123 & 0.000165 & 0.000565 & 0.001279 \\
\hline 153.75 & -0.00125 & 0.000152 & 0.00058 & 0.00129 \\
\hline 154.50 & -0.00124 & 0.000168 & 0.000582 & 0.001281 \\
\hline 155.25 & -0.00124 & 0.000157 & 0.000584 & 0.001271 \\
\hline 156.00 & -0.00124 & 0.000158 & 0.000581 & 0.001307 \\
\hline 156.75 & -0.00126 & 0.000151 & 0.000582 & 0.0013 \\
\hline 157.50 & -0.00127 & 0.000146 & 0.000577 & 0.001258 \\
\hline 158.25 & -0.00125 & 0.000151 & 0.000591 & 0.001312 \\
\hline 159.00 & -0.00125 & 0.000141 & 0.000586 & 0.001278 \\
\hline 159.75 & -0.00128 & 0.000136 & 0.000591 & 0.001301 \\
\hline 160.50 & -0.00129 & 0.000104 & 0.000591 & 0.001325 \\
\hline 161.25 & -0.00129 & 0.000147 & 0.000585 & 0.001301 \\
\hline 162.00 & -0.00127 & 0.000113 & 0.000594 & 0.001318 \\
\hline 162.75 & -0.00128 & 0.000112 & 0.00059 & 0.001314 \\
\hline 163.50 & -0.0013 & $9.11 \mathrm{E}-05$ & 0.000592 & 0.0013 \\
\hline 164.25 & -0.00128 & 0.000114 & 0.000603 & 0.001326 \\
\hline 165.00 & -0.00129 & $9.62 \mathrm{E}-05$ & 0.000591 & 0.001307 \\
\hline 165.75 & -0.0013 & 8.87E-05 & 0.000598 & 0.001334 \\
\hline 166.50 & -0.0013 & 0.000105 & 0.000594 & 0.00131 \\
\hline 167.25 & -0.00128 & $9.68 \mathrm{E}-05$ & 0.000604 & 0.001327 \\
\hline 168.00 & -0.00129 & $9.86 \mathrm{E}-05$ & 0.000602 & 0.00132 \\
\hline 168.75 & -0.00133 & 8.06E-05 & 0.000582 & 0.001322 \\
\hline 169.50 & -0.00131 & 0.000106 & 0.000615 & 0.001336 \\
\hline 170.25 & -0.00132 & 9.47E-05 & 0.000611 & 0.001334 \\
\hline 171.00 & -0.00131 & $9.25 \mathrm{E}-05$ & 0.000617 & 0.001333 \\
\hline 171.75 & -0.00131 & 8.93E-05 & 0.000633 & 0.001344 \\
\hline 172.50 & -0.0013 & $7.62 \mathrm{E}-05$ & 0.000621 & 0.001339 \\
\hline 173.25 & -0.00132 & $7.68 \mathrm{E}-05$ & 0.000632 & 0.001338 \\
\hline 174.00 & -0.00132 & 3.39E-05 & 0.000616 & 0.001353 \\
\hline 174.75 & -0.00134 & 4.74E-05 & 0.000619 & 0.001384 \\
\hline 175.50 & -0.00132 & 6.87E-05 & 0.000625 & 0.001341 \\
\hline 176.25 & -0.00134 & $5.82 \mathrm{E}-05$ & 0.000618 & 0.001373 \\
\hline 177.00 & -0.00134 & 5.35E-05 & 0.000619 & 0.001365 \\
\hline
\end{tabular}




\begin{tabular}{|c|c|c|c|c|}
\hline 177.75 & -0.00133 & 4.98E-05 & 0.000613 & 0.001346 \\
\hline 178.50 & -0.00135 & $5.94 \mathrm{E}-05$ & 0.000617 & 0.001353 \\
\hline 179.25 & -0.00134 & 5.13E-05 & 0.000625 & 0.001367 \\
\hline 180.00 & -0.00135 & $3.74 \mathrm{E}-05$ & 0.000638 & 0.001359 \\
\hline 180.75 & -0.00134 & 5.03E-05 & 0.000627 & 0.001361 \\
\hline 181.50 & -0.00135 & 2.63E-05 & 0.000623 & 0.001342 \\
\hline 182.25 & -0.00137 & $4.28 \mathrm{E}-05$ & 0.000642 & 0.001389 \\
\hline 183.00 & -0.00136 & 4.1E-05 & 0.000633 & 0.001372 \\
\hline 183.75 & -0.00136 & $2.16 \mathrm{E}-05$ & 0.000635 & 0.001383 \\
\hline 184.50 & -0.00138 & $1.68 \mathrm{E}-05$ & 0.000636 & 0.00135 \\
\hline 185.25 & -0.00136 & $6.1 \mathrm{E}-05$ & 0.000643 & 0.001358 \\
\hline 186.00 & -0.00136 & $3.5 \mathrm{E}-05$ & 0.000654 & 0.001402 \\
\hline 186.75 & -0.00136 & 4.61E-05 & 0.000624 & 0.001343 \\
\hline 187.50 & -0.00135 & 4.67E-05 & 0.000632 & 0.001398 \\
\hline 188.25 & -0.00137 & 2.31E-05 & 0.000634 & 0.001371 \\
\hline 189.00 & -0.00139 & $1.66 \mathrm{E}-05$ & 0.000646 & 0.001391 \\
\hline 189.75 & -0.00138 & $4.05 \mathrm{E}-05$ & 0.000646 & 0.001412 \\
\hline 190.50 & -0.00134 & 4.12E-05 & 0.000642 & 0.00138 \\
\hline 191.25 & -0.00136 & $3.55 \mathrm{E}-05$ & 0.000643 & 0.001386 \\
\hline 192.00 & -0.00139 & $1.39 \mathrm{E}-05$ & 0.000646 & 0.001395 \\
\hline 192.75 & -0.00136 & $2.84 \mathrm{E}-05$ & 0.000639 & 0.001376 \\
\hline 193.50 & -0.00139 & $1.78 \mathrm{E}-05$ & 0.000655 & 0.00138 \\
\hline 194.25 & -0.00138 & $2.31 \mathrm{E}-05$ & 0.000648 & 0.001391 \\
\hline 195.00 & -0.00139 & 9.31E-06 & 0.000649 & 0.001389 \\
\hline 195.75 & -0.00139 & $-1.2 \mathrm{E}-05$ & 0.00064 & 0.001387 \\
\hline 196.50 & -0.00138 & 1.01E-05 & 0.000654 & 0.001393 \\
\hline 197.25 & -0.00139 & $6.49 \mathrm{E}-06$ & 0.000641 & 0.001402 \\
\hline 198.00 & -0.00137 & 1.99E-05 & 0.000661 & 0.001417 \\
\hline 198.75 & -0.00139 & $-5.3 E-06$ & 0.000644 & 0.001372 \\
\hline 199.50 & -0.00142 & $-1.5 \mathrm{E}-05$ & 0.000654 & 0.001405 \\
\hline 200.25 & -0.00138 & $4.75 \mathrm{E}-06$ & 0.000652 & 0.001387 \\
\hline 201.00 & -0.00139 & $-1.2 \mathrm{E}-06$ & 0.00065 & 0.001385 \\
\hline 201.75 & -0.00141 & $-9.6 \mathrm{E}-06$ & 0.000661 & 0.001432 \\
\hline 202.50 & -0.0014 & $-7.8 \mathrm{E}-06$ & 0.000676 & 0.001438 \\
\hline 203.25 & -0.00139 & $1.3 \mathrm{E}-05$ & 0.000654 & 0.001398 \\
\hline 204.00 & -0.00139 & $-1.2 \mathrm{E}-05$ & 0.000666 & 0.001408 \\
\hline 204.75 & -0.00139 & 9.91E-06 & 0.000645 & 0.00139 \\
\hline 205.50 & -0.0014 & $-8.8 \mathrm{E}-06$ & 0.000642 & 0.001392 \\
\hline 206.25 & -0.00139 & $6.24 \mathrm{E}-06$ & 0.00066 & 0.001437 \\
\hline 207.00 & -0.0014 & $-8.3 E-06$ & 0.000658 & 0.001422 \\
\hline 207.75 & -0.00142 & $-2 \mathrm{E}-05$ & 0.000654 & 0.001393 \\
\hline 208.50 & -0.00141 & $-1.9 E-05$ & 0.000665 & 0.001408 \\
\hline 209.25 & -0.00138 & 2.37E-05 & 0.000651 & 0.001437 \\
\hline 210.00 & -0.00142 & $1.01 \mathrm{E}-05$ & 0.000656 & 0.001417 \\
\hline 210.75 & -0.00143 & $-1.8 \mathrm{E}-05$ & 0.000658 & 0.001395 \\
\hline 211.50 & -0.0014 & $-1.7 \mathrm{E}-05$ & 0.000672 & 0.001426 \\
\hline 212.25 & -0.00138 & $-3.5 E-07$ & 0.000663 & 0.001415 \\
\hline 213.00 & -0.00141 & 1.65E-05 & 0.000665 & 0.001405 \\
\hline
\end{tabular}




$\begin{array}{lllll}213.75 & -0.00141 & -2.9 \mathrm{E}-05 & 0.000648 & 0.001381 \\ 214.50 & -0.00141 & -7.1 \mathrm{E}-06 & 0.000763 & 0.001512 \\ 215.25 & -0.00141 & -2 \mathrm{E}-05 & 0.000757 & 0.00151 \\ 216.00 & -0.00141 & 8.99 \mathrm{E}-06 & 0.000761 & 0.001496 \\ 216.75 & -0.00141 & -1.9 \mathrm{E}-05 & 0.000763 & 0.001521 \\ 217.50 & -0.00141 & -5.9 \mathrm{E}-06 & 0.000767 & 0.001508 \\ 218.25 & -0.00139 & -7.8 \mathrm{E}-06 & 0.000882 & 0.001612 \\ 219.00 & -0.00141 & -4.2 \mathrm{E}-05 & 0.000877 & 0.001628 \\ 219.75 & -0.00141 & -1.8 \mathrm{E}-05 & 0.000887 & 0.001618 \\ 220.50 & -0.0014 & 6.67 \mathrm{E}-06 & 0.000893 & 0.001643 \\ 221.25 & -0.00139 & 8.98 \mathrm{E}-06 & 0.000887 & 0.001637\end{array}$




\section{Appendix 4 - Data for figures 5, S11 and S12}

\begin{tabular}{|c|c|c|c|c|}
\hline time / min & $\begin{array}{c}\text { raw peak at } \\
862 \mathrm{~cm}^{-1}\end{array}$ & $\begin{array}{c}\text { normalised peak } \\
\text { at } 862 \mathrm{~cm}^{-1}\end{array}$ & $\begin{array}{c}\text { raw peak at } \\
1036 \mathrm{~cm}^{-1}\end{array}$ & $\begin{array}{c}\text { normalised peak } \\
\text { at } 1036 \mathrm{~cm}^{-1}\end{array}$ \\
\hline 0.00 & -0.0013 & $-7.36 \mathrm{E}-06$ & -0.00112 & 0.0004683 \\
\hline 0.75 & -0.00128 & $1.28 \mathrm{E}-05$ & -0.00111 & 0.0004510 \\
\hline 1.50 & -0.00129 & $6.96 \mathrm{E}-06$ & -0.00113 & 0.0004048 \\
\hline 2.25 & -0.00131 & $-1.32 \mathrm{E}-05$ & -0.00113 & 0.0004695 \\
\hline 3.00 & -0.00129 & 8.31E-07 & -0.00112 & 0.0004366 \\
\hline 3.75 & -0.00067 & $6.26 \mathrm{E}-04$ & -0.00103 & 0.0002707 \\
\hline 4.50 & -0.00062 & $6.75 \mathrm{E}-04$ & -0.00101 & 0.0002629 \\
\hline 5.25 & -0.00065 & $6.42 \mathrm{E}-04$ & -0.001 & 0.0002467 \\
\hline 6.00 & -0.00061 & $6.89 \mathrm{E}-04$ & -0.001 & 0.0002455 \\
\hline 6.75 & -0.00063 & $6.65 \mathrm{E}-04$ & -0.00099 & 0.0002401 \\
\hline 7.50 & 0.00018 & $1.48 \mathrm{E}-03$ & -0.00086 & -0.0000158 \\
\hline 8.25 & 0.000175 & $1.47 \mathrm{E}-03$ & -0.00087 & -0.0000076 \\
\hline 9.00 & 0.000164 & $1.46 \mathrm{E}-03$ & -0.00085 & 0.0000154 \\
\hline 9.75 & 0.000165 & $1.47 \mathrm{E}-03$ & -0.00085 & 0.0000204 \\
\hline 10.50 & 0.00016 & $1.46 \mathrm{E}-03$ & -0.00087 & -0.0000124 \\
\hline 11.25 & 0.000106 & $1.41 \mathrm{E}-03$ & -0.00084 & 0.0000298 \\
\hline 12.00 & $9.01 \mathrm{E}-05$ & $1.39 \mathrm{E}-03$ & -0.00082 & 0.0000573 \\
\hline 12.75 & $6.94 \mathrm{E}-05$ & $1.37 \mathrm{E}-03$ & -0.00083 & 0.0000457 \\
\hline 13.50 & $4.75 E-05$ & $1.35 \mathrm{E}-03$ & -0.00083 & 0.0000425 \\
\hline 14.25 & $2.58 \mathrm{E}-05$ & $1.32 \mathrm{E}-03$ & -0.00083 & 0.0000398 \\
\hline 15.00 & $5.25 \mathrm{E}-05$ & $1.35 \mathrm{E}-03$ & -0.00082 & 0.0000805 \\
\hline 15.75 & $4.15 \mathrm{E}-05$ & $1.34 \mathrm{E}-03$ & -0.0008 & 0.0001021 \\
\hline 16.50 & $3.44 \mathrm{E}-05$ & $1.33 \mathrm{E}-03$ & -0.00081 & 0.0001043 \\
\hline 17.25 & $3.62 \mathrm{E}-05$ & $1.33 \mathrm{E}-03$ & -0.00081 & 0.0001051 \\
\hline 18.00 & $2.31 \mathrm{E}-05$ & $1.32 \mathrm{E}-03$ & -0.00081 & 0.0000935 \\
\hline 18.75 & $2.94 \mathrm{E}-06$ & $1.30 \mathrm{E}-03$ & -0.0008 & 0.0001124 \\
\hline 19.50 & $-9.13 E-06$ & $1.29 \mathrm{E}-03$ & -0.00081 & 0.0001084 \\
\hline 20.25 & $1.35 \mathrm{E}-05$ & $1.31 \mathrm{E}-03$ & -0.00081 & 0.0001205 \\
\hline 21.00 & $-1.36 \mathrm{E}-05$ & $1.28 \mathrm{E}-03$ & -0.0008 & 0.0001346 \\
\hline 21.75 & $-1.36 \mathrm{E}-05$ & $1.29 \mathrm{E}-03$ & -0.00079 & 0.0001631 \\
\hline 22.50 & $-5.40 \mathrm{E}-06$ & $1.29 \mathrm{E}-03$ & -0.00077 & 0.0001964 \\
\hline 23.25 & $-4.35 E-05$ & $1.26 \mathrm{E}-03$ & -0.00079 & 0.0001481 \\
\hline 24.00 & $-1.93 E-05$ & $1.28 \mathrm{E}-03$ & -0.00078 & 0.0001957 \\
\hline 24.75 & $-5.54 \mathrm{E}-05$ & $1.24 \mathrm{E}-03$ & -0.00078 & 0.0001688 \\
\hline 25.50 & $-6.89 E-05$ & $1.23 \mathrm{E}-03$ & -0.00078 & 0.0001692 \\
\hline 26.25 & $-7.64 \mathrm{E}-05$ & $1.22 \mathrm{E}-03$ & -0.00078 & 0.0001981 \\
\hline 27.00 & $-6.42 \mathrm{E}-05$ & $1.24 \mathrm{E}-03$ & -0.00078 & 0.0001853 \\
\hline 27.75 & $-7.81 \mathrm{E}-05$ & $1.22 \mathrm{E}-03$ & -0.00076 & 0.0002314 \\
\hline 28.50 & -0.0001 & 1.19E-03 & -0.00076 & 0.0002426 \\
\hline 29.25 & $-7.65 E-05$ & $1.22 \mathrm{E}-03$ & -0.00076 & 0.0002131 \\
\hline 30.00 & -0.0001 & $1.20 \mathrm{E}-03$ & -0.00076 & 0.0002320 \\
\hline 30.75 & $-9.03 E-05$ & $1.21 \mathrm{E}-03$ & -0.00075 & 0.0002363 \\
\hline 31.50 & -0.00011 & 1.19E-03 & -0.00076 & 0.0002338 \\
\hline 32.25 & -0.00014 & $1.16 \mathrm{E}-03$ & -0.00075 & 0.0002453 \\
\hline 33.00 & -0.00013 & 1.17E-03 & -0.00074 & 0.0002526 \\
\hline
\end{tabular}




\begin{tabular}{|c|c|c|c|c|}
\hline 33.75 & -0.00013 & 1.17E-03 & -0.00075 & 0.0002626 \\
\hline 34.50 & -0.00013 & 1.17E-03 & -0.00074 & 0.0002857 \\
\hline 35.25 & -0.00017 & $1.13 \mathrm{E}-03$ & -0.00075 & 0.0002798 \\
\hline 36.00 & -0.00018 & $1.12 \mathrm{E}-03$ & -0.00075 & 0.0002578 \\
\hline 36.75 & -0.00017 & $1.13 \mathrm{E}-03$ & -0.00075 & 0.0002605 \\
\hline 37.50 & -0.00016 & $1.13 \mathrm{E}-03$ & -0.00074 & 0.0002867 \\
\hline 38.25 & -0.00019 & $1.11 \mathrm{E}-03$ & -0.00072 & 0.0002842 \\
\hline 39.00 & -0.00019 & $1.11 \mathrm{E}-03$ & -0.00074 & 0.0003155 \\
\hline 39.75 & -0.00018 & $1.12 \mathrm{E}-03$ & -0.00074 & 0.0003216 \\
\hline 40.50 & -0.00019 & $1.11 \mathrm{E}-03$ & -0.00074 & 0.0003221 \\
\hline 41.25 & -0.0002 & 1.10E-03 & -0.00073 & 0.0002923 \\
\hline 42.00 & -0.00019 & 1.10E-03 & -0.00073 & 0.0003345 \\
\hline 42.75 & -0.00021 & $1.09 \mathrm{E}-03$ & -0.00071 & 0.0003897 \\
\hline 43.50 & -0.00021 & $1.08 \mathrm{E}-03$ & -0.00073 & 0.0003242 \\
\hline 44.25 & -0.00025 & $1.04 \mathrm{E}-03$ & -0.00073 & 0.0003479 \\
\hline 45.00 & -0.00024 & $1.06 \mathrm{E}-03$ & -0.00072 & 0.0003214 \\
\hline 45.75 & -0.00024 & $1.06 \mathrm{E}-03$ & -0.0007 & 0.0003759 \\
\hline 46.50 & -0.00023 & $1.06 \mathrm{E}-03$ & -0.00071 & 0.0003737 \\
\hline 47.25 & -0.00024 & $1.06 \mathrm{E}-03$ & -0.00071 & 0.0003923 \\
\hline 48.00 & -0.00025 & $1.05 \mathrm{E}-03$ & -0.00069 & 0.0003791 \\
\hline 48.75 & -0.00029 & $1.01 \mathrm{E}-03$ & -0.00071 & 0.0003897 \\
\hline 49.50 & -0.00027 & $1.03 \mathrm{E}-03$ & -0.00072 & 0.0003660 \\
\hline 50.25 & -0.00028 & $1.02 \mathrm{E}-03$ & -0.0007 & 0.0004131 \\
\hline 51.00 & -0.00029 & $1.01 \mathrm{E}-03$ & -0.0007 & 0.0004004 \\
\hline 51.75 & -0.00025 & $1.05 \mathrm{E}-03$ & -0.0007 & 0.0004009 \\
\hline 52.50 & -0.00028 & $1.02 \mathrm{E}-03$ & -0.00068 & 0.0003989 \\
\hline 53.25 & -0.00028 & $1.02 \mathrm{E}-03$ & -0.00069 & 0.0004431 \\
\hline 54.00 & -0.00026 & $1.04 \mathrm{E}-03$ & -0.00069 & 0.0004191 \\
\hline 54.75 & -0.0003 & $1.00 \mathrm{E}-03$ & -0.00069 & 0.0004384 \\
\hline 55.50 & -0.0003 & $9.94 \mathrm{E}-04$ & -0.0007 & 0.0004115 \\
\hline 56.25 & -0.00029 & $1.01 \mathrm{E}-03$ & -0.00068 & 0.0004251 \\
\hline 57.00 & -0.0003 & $9.99 \mathrm{E}-04$ & -0.00069 & 0.0004621 \\
\hline 57.75 & -0.00029 & $1.01 \mathrm{E}-03$ & -0.00069 & 0.0004408 \\
\hline 58.50 & -0.00034 & $9.54 \mathrm{E}-04$ & -0.00067 & 0.0004638 \\
\hline 59.25 & -0.00033 & $9.71 \mathrm{E}-04$ & -0.00069 & 0.0004466 \\
\hline 60.00 & -0.00033 & $9.70 \mathrm{E}-04$ & -0.00068 & 0.0004735 \\
\hline 60.75 & -0.00034 & $9.58 \mathrm{E}-04$ & -0.00068 & 0.0004501 \\
\hline 61.50 & -0.00037 & $9.26 \mathrm{E}-04$ & -0.00069 & 0.0004805 \\
\hline 62.25 & -0.00036 & $9.41 \mathrm{E}-04$ & -0.00068 & 0.0004421 \\
\hline 63.00 & -0.00034 & $9.60 \mathrm{E}-04$ & -0.00068 & 0.0004787 \\
\hline 63.75 & -0.00036 & $9.35 \mathrm{E}-04$ & -0.00068 & 0.0004683 \\
\hline 64.50 & -0.00038 & $9.22 \mathrm{E}-04$ & -0.00067 & 0.0004714 \\
\hline 65.25 & -0.00038 & $9.13 \mathrm{E}-04$ & -0.00067 & 0.0005135 \\
\hline 66.00 & -0.00036 & $9.40 \mathrm{E}-04$ & -0.00067 & 0.0004796 \\
\hline 66.75 & -0.00037 & $9.23 \mathrm{E}-04$ & -0.00066 & 0.0004848 \\
\hline 67.50 & -0.00036 & $9.40 \mathrm{E}-04$ & -0.00068 & 0.0004877 \\
\hline 68.25 & -0.00041 & 8.89E-04 & -0.00066 & 0.0005511 \\
\hline 69.00 & -0.00041 & $8.85 \mathrm{E}-04$ & -0.00068 & 0.0005064 \\
\hline
\end{tabular}




\begin{tabular}{|c|c|c|c|c|}
\hline 69.75 & -0.0004 & $8.94 \mathrm{E}-04$ & -0.00065 & 0.0005287 \\
\hline 70.50 & -0.00043 & $8.71 \mathrm{E}-04$ & -0.00066 & 0.0005172 \\
\hline 71.25 & -0.0004 & $9.02 E-04$ & -0.00067 & 0.0004925 \\
\hline 72.00 & -0.00038 & 9.19E-04 & -0.00065 & 0.0005208 \\
\hline 72.75 & -0.00043 & 8.67E-04 & -0.00064 & 0.0005362 \\
\hline 73.50 & -0.00042 & 8.71E-04 & -0.00066 & 0.0005203 \\
\hline 74.25 & -0.00042 & $8.78 \mathrm{E}-04$ & -0.00065 & 0.0005333 \\
\hline 75.00 & -0.00044 & 8.55E-04 & -0.00067 & 0.0005224 \\
\hline 75.75 & -0.00045 & $8.48 \mathrm{E}-04$ & -0.00064 & 0.0005438 \\
\hline 76.50 & -0.00043 & $8.63 \mathrm{E}-04$ & -0.00065 & 0.0005370 \\
\hline 77.25 & -0.00046 & 8.37E-04 & -0.00065 & 0.0005553 \\
\hline 78.00 & -0.00044 & 8.57E-04 & -0.00064 & 0.0005702 \\
\hline 78.75 & -0.00042 & 8.81E-04 & -0.00066 & 0.0005317 \\
\hline 79.50 & -0.00045 & $8.49 \mathrm{E}-04$ & -0.00065 & 0.0005626 \\
\hline 80.25 & -0.00048 & $8.18 \mathrm{E}-04$ & -0.00065 & 0.0005587 \\
\hline 81.00 & -0.00045 & 8.52E-04 & -0.00064 & 0.0005513 \\
\hline 81.75 & -0.00047 & $8.23 \mathrm{E}-04$ & -0.00063 & 0.0005877 \\
\hline 82.50 & -0.00046 & 8.37E-04 & -0.00064 & 0.0005706 \\
\hline 83.25 & -0.00047 & 8.30E-04 & -0.00066 & 0.0005752 \\
\hline 84.00 & -0.00047 & 8.29E-04 & -0.00065 & 0.0005539 \\
\hline 84.75 & -0.00049 & 8.07E-04 & -0.00066 & 0.0005419 \\
\hline 85.50 & -0.00046 & 8.39E-04 & -0.00064 & 0.0005571 \\
\hline 86.25 & -0.00047 & $8.28 \mathrm{E}-04$ & -0.00063 & 0.0005683 \\
\hline 87.00 & -0.00049 & $8.05 E-04$ & -0.00063 & 0.0006188 \\
\hline 87.75 & -0.00051 & 7.93E-04 & -0.00064 & 0.0005894 \\
\hline 88.50 & -0.00047 & 8.31E-04 & -0.00065 & 0.0005864 \\
\hline 89.25 & -0.00051 & $7.89 E-04$ & -0.00064 & 0.0005814 \\
\hline 90.00 & -0.00049 & $8.06 \mathrm{E}-04$ & -0.00064 & 0.0005822 \\
\hline 90.75 & -0.00049 & 8.04E-04 & -0.00063 & 0.0006274 \\
\hline 91.50 & -0.00045 & $8.43 \mathrm{E}-04$ & -0.00064 & 0.0005720 \\
\hline 92.25 & -0.00048 & $8.14 \mathrm{E}-04$ & -0.00064 & 0.0005831 \\
\hline 93.00 & -0.00049 & $8.04 \mathrm{E}-04$ & -0.00063 & 0.0006109 \\
\hline 93.75 & -0.00048 & $8.16 \mathrm{E}-04$ & -0.00062 & 0.0006168 \\
\hline 94.50 & -0.00051 & 7.90E-04 & -0.00062 & 0.0006111 \\
\hline 95.25 & -0.0005 & 7.95E-04 & -0.00063 & 0.0005970 \\
\hline 96.00 & -0.00052 & 7.77E-04 & -0.00063 & 0.0006364 \\
\hline 96.75 & -0.00054 & 7.54E-04 & -0.00062 & 0.0006305 \\
\hline 97.50 & -0.00052 & 7.80E-04 & -0.00062 & 0.0006492 \\
\hline 98.25 & -0.00054 & $7.53 \mathrm{E}-04$ & -0.00062 & 0.0006529 \\
\hline 99.00 & -0.00053 & $7.72 \mathrm{E}-04$ & -0.00062 & 0.0006239 \\
\hline 99.75 & -0.00051 & $7.86 \mathrm{E}-04$ & -0.00064 & 0.0006367 \\
\hline 100.50 & -0.00053 & 7.66E-04 & -0.00062 & 0.0006146 \\
\hline 101.25 & -0.00054 & 7.56E-04 & -0.00062 & 0.0006328 \\
\hline 102.00 & -0.00051 & 7.90E-04 & -0.00062 & 0.0006416 \\
\hline 102.75 & -0.00056 & 7.41E-04 & -0.00062 & 0.0006302 \\
\hline 103.50 & -0.00055 & 7.51E-04 & -0.00061 & 0.0006416 \\
\hline 104.25 & -0.00053 & 7.68E-04 & -0.00062 & 0.0006500 \\
\hline 105.00 & -0.00056 & 7.41E-04 & -0.00063 & 0.0006359 \\
\hline
\end{tabular}




$\begin{array}{lllll}105.75 & -0.00053 & 7.69 \mathrm{E}-04 & -0.00062 & 0.0006321 \\ 106.50 & -0.00053 & 7.68 \mathrm{E}-04 & -0.00061 & 0.0006673 \\ 107.25 & -0.00062 & 6.78 \mathrm{E}-04 & -0.00047 & 0.0008266 \\ 108.00 & -0.0006 & 6.94 \mathrm{E}-04 & -0.00047 & 0.0008203 \\ 108.75 & -0.00065 & 6.51 \mathrm{E}-04 & -0.00046 & 0.0008172 \\ 109.50 & -0.00061 & 6.82 \mathrm{E}-04 & -0.00048 & 0.0008120 \\ 110.25 & -0.00062 & 6.76 \mathrm{E}-04 & -0.00046 & 0.0008291 \\ 111.00 & -0.00071 & 5.91 \mathrm{E}-04 & -0.00028 & 0.0010254 \\ 111.75 & -0.00072 & 5.76 \mathrm{E}-04 & -0.00025 & 0.0010557 \\ 112.50 & -0.00071 & 5.86 \mathrm{E}-04 & -0.00027 & 0.0010185 \\ 113.25 & -0.00073 & 5.70 \mathrm{E}-04 & -0.00028 & 0.0010145 \\ 114.00 & -0.00071 & 5.90 \mathrm{E}-04 & -0.00027 & 0.0010340\end{array}$

